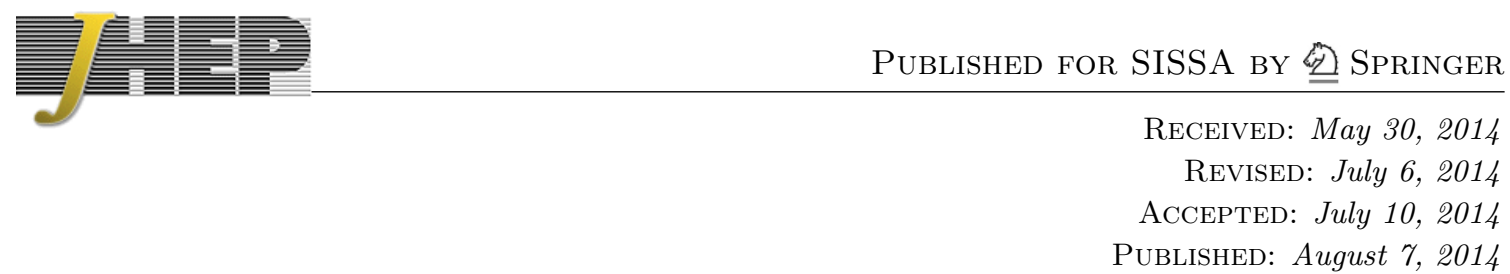

\title{
Entropy current for non-relativistic fluid
}

\author{
Nabamita Banerjee, ${ }^{a}$ Suvankar Dutta, ${ }^{b}$ Akash Jain $^{b}$ and Dibakar Roychowdhury ${ }^{b}$ \\ ${ }^{a}$ Indian Association for the Cultivation of Science, \\ Jadavpur, Kolkata, India \\ ${ }^{b}$ Dept. of Physics, Indian Institute of Science Education and Research Bhopal, \\ Bhopal, India \\ E-mail: tpnb@iacs.res.in, suvankar@iiserb.ac.in, \\ ajainphysics@gmail.com, dibakarphys@gmail.com
}

ABSTRACT: We study transport properties of a parity-odd, non-relativistic charged fluid in presence of background electric and magnetic fields. To obtain stress tensor and charged current for the non-relativistic system we start with the most generic relativistic fluid, living in one higher dimension and reduce the constituent equations along the light-cone direction. We also reduce the equation satisfied by the entropy current of the relativistic theory and obtain a consistent entropy current for the non-relativistic system (we call it "canonical form" of the entropy current). Demanding that the non-relativistic fluid satisfies the second law of thermodynamics we impose constraints on various first order transport coefficients. For parity even fluid, this is straight forward; it tells us positive definiteness of different transport coefficients like viscosity, thermal conductivity, electric conductivity etc. However for parity-odd fluid, canonical form of the entropy current fails to confirm the second law of thermodynamics. Therefore, we need to add two parity-odd vectors to the entropy current with arbitrary coefficients. Upon demanding the validity of second law, we see that one can fix these two coefficients exactly.

KeYwords: Quantum Dissipative Systems, Anomalies in Field and String Theories

ARXiv EPRINT: 1405.5687 


\section{Contents}

1 Introduction and summary 1

2 Relativistic anomalous charged fluid $\quad 4$

2.1 Anomalous charged fluid: energy-momentum tensor and current 6

$\begin{array}{lll}2.2 & \text { Entropy current } & 8\end{array}$

3 Non-relativistic charged fluid $\quad 9$

3.1 Non-relativistic dissipative fluid in background electromagnetic field $\quad 10$

4 Light-cone reduction $\quad 11$

$\begin{array}{lll}4.1 & \text { Reduction of background fields } & 13\end{array}$

4.2 Reduction of energy-momentum tensor and charge current 14

$\begin{array}{lll}\text { 4.2.1 } & T^{++} \text {calculation } & 15\end{array}$

$\begin{array}{lll}4.2 .2 & T^{i+} \text { calculation } & 15\end{array}$

$\begin{array}{lll}4.2 .3 & T^{i j} \text { calculation } & 15\end{array}$

$\begin{array}{lll}\text { 4.2.4 } & T^{+-} \text {calculation } & 16\end{array}$

$\begin{array}{lll}4.2 .5 & T^{i-} \text { calculation } & 16\end{array}$

$\begin{array}{lll}4.2 .6 & J^{+} \text {calculation } & 17\end{array}$

$\begin{array}{lll}4.2 .7 & J^{i} \text { calculation } & 17\end{array}$

$\begin{array}{lll}4.3 & \text { Thermodynamics of the reduced system } & 19\end{array}$

5 Non-relativistic entropy current $\quad 20$

5.1 Parity-odd corrections to NR entropy current 22

5.2 Incompressible fluid in constant magnetic background in (2+1)-dim 24

$\begin{array}{llr}6 & \text { Discussion } & 25\end{array}$

$\begin{array}{ll}\text { A Magnetic limit of electrodynamics } & 27\end{array}$

$\begin{array}{lll}\text { B } 1 / \text { c expansion of relativistic fluid dynamics } & 28\end{array}$

B.1 1/c expansion of thermodynamics 30

\section{Introduction and summary}

Hydrodynamics is an effective description of nearly equilibrium interacting many body system. A fluid system is considered to be continuous, i.e. when we talk about a small volume element (or 'fluid particle') of the fluid, it still contains a large number of constituent particles (atoms/molecules). More specifically, the size of the fluid particle is much much greater than the mean free path of the system. The equations of hydrodynamics assume 
that the fluid is in local thermodynamic equilibrium at each point in space and time, ${ }^{1}$ even though different thermodynamic quantities like fluid velocity $\vec{v}(\vec{x}, t)$, energy density $\epsilon(\vec{x}, t)$, pressure $p(\vec{x}, t)$, fluid density $\rho(\vec{x}, t)$, temperature $\tau(\vec{x}, t)$ etc. may vary. Fluid mechanics applies only when the length scales of variation of thermodynamic variables are large compared to equilibration length scale of the fluid, namely the mean free path [1].

Although hydrodynamics is an old and well-studied subject in physics, recently there has been much interest and progress in relativistic, charged, viscous fluid in presence of some global anomaly. The first evidence of relativistic anomalous fluids was observed in [2,3], in the context of the $A d S / C F T$ correspondence. In these papers the authors found a new term (and hence a new transport coefficient) in the charge current in presence of a ChernSimons term in the bulk Lagrangian. Later, in [4] it has been shown that this kind of term in charge current is not only allowed by the symmetry but is required by triangle anomalies and the second law of thermodynamics. Demanding the positivity of local entropy current they showed that the coefficient of this new term is also fixed in terms of anomaly coefficient of the theory. In general, the second law of thermodynamics (or equivalently, positivity of local entropy current) imposes restrictions on different transport coefficients. Interested readers can look at [5-12].

This was about relativistic fluids. Much attention has not been paid in parity-odd charged non-relativistic fluid in presence of background electromagnetic fields. In [13] an attempt has been made to study non-relativistic neutral fluids as a consistent light-cone reduction of relativistic fluid systems. In [14], the idea has been extended to charged fluid without any background field. However, main focus of these two papers was to construct the holographic duals of long wave length fluctuations (hydrodynamic limit) of conformal non-relativistic field theories. They also computed few transport coefficients (for example thermal conductivity) holographically.

Recently in [15], non-relativistic, parity-odd, first order charged fluid in 2 dimensions has been discussed. The authors started with a $2+1$ dimensional relativistic, parity-odd charged fluid in presence of background gauge fields and took suitable non-relativistic limit of the system. They identified the parity-violating contributions to the non-relativistic constitutive relations, which include the Hall current flowing perpendicular to the temperature gradient, the Hall viscosity and the Leduc-Righi energy current. ${ }^{2}$ However, in these papers, much attention has not been paid to second law of thermodynamics. It is well known that the local second law of thermodynamics imposes several constraints on the flow and the transport coefficients. For example, the local second law demands that shear, bulk viscosity coefficients and thermal conductivity have to be positive definite for a non-relativistic fluid [1]. This particular issue, for relativistic fluid, has been discussed vividly in various papers mentioned above. However, the constraints from second law of thermodynamics are not yet well understood for a general class of non-relativistic fluids (charged fluid in presence of background fields). Goal of this paper is to write down a local entropy current, which satisfies second law of thermodynamics, for a non-relativistic

\footnotetext{
${ }^{1}$ The modern understanding of hydrodynamics however, suggests that it starts to apply long before the absolute equilibrium is reached.

${ }^{2}$ See also [16].
} 
charged fluid in presence of arbitrary background electromagnetic field and to study the constraints on the transport coefficients.

One way to obtain the energy momentum tensor, charge current and entropy current for a non-relativistic theory is to reduce the constitutive equations and entropy current equation of a relativistic fluid over a light-cone direction. We start with a $(3+1)$-dimensional relativistic charged fluid with global $\mathrm{U}(1)^{3}$ anomaly and write down their non-relativistic counterparts. We follow the light-cone reduction technique, which was introduced in [13], and shall as well review it in our paper. Let us conclude this section with an outline and summary of our main results.

\section{Outline and summary.}

- In section 2 we consider first order, relativistic, charged anomalous fluid in $(3+1)$ dimensions in presence of background electromagnetic fields. We briefly review the work of [4] and show how parity-odd transport coefficients are related to the coefficient of anomaly from the positivity of divergence of local entropy current.

- In section 3 we highlight the important equations of non-relativistic hydrodynamic system.

- In section 4 we consistently obtain the energy current density $\left(j^{i}\right)$, stress tensor $\left(t^{i j}\right)$ and charge current density $\left(j_{I}^{i}\right)$ of non-relativistic fluid by Light Cone Reduction (LCR). We also find different non-relativistic fluid variables (thermodynamic quantities and transport coefficients) in terms of the relativistic fluid variables. Here we mention the final expression for stress tensor, energy current and charge current: ${ }^{3}$

$$
\begin{aligned}
& t^{i j}= v^{i} v^{j} \rho+p g^{i j}-n \sigma^{i j}-z g^{i j} \nabla_{k} v^{k} \\
& j^{i}=v^{i}\left(\epsilon+p+\frac{1}{2} \rho \mathbf{v}^{2}\right)-n \sigma^{i k} v_{k}-z v^{i} \nabla_{k} v^{k}-\kappa \nabla^{i} \tau-\tau \sigma_{I} \nabla^{i}\left(\frac{\mu_{I}}{\tau}\right)+\sigma_{I}\left(\epsilon_{I}^{i}-v_{j} \beta_{I}^{j i}\right) \\
& j_{I}^{i}=q_{I} v^{i}-\tilde{\kappa}_{I} \nabla^{i} \tau-\tilde{\xi}_{I J} \nabla^{i}\left(\frac{\mu_{J}}{\tau}\right)-\tilde{m}_{I} \nabla^{i} p \\
&+\tilde{\sigma}_{I J}\left(\epsilon_{J}^{i}-v_{k} \beta_{J}^{k i}\right)+\left\{\bar{\kappa}_{I} \epsilon^{i j} \nabla_{j} \tau+\bar{\xi}_{I J} \epsilon^{i j} \nabla_{j}\left(\frac{\mu_{J}}{\tau}\right)\right. \\
&\left.-\bar{m}_{I} \epsilon^{i j} \nabla_{j} p+\bar{\sigma}_{I J} \epsilon^{i j}\left(\epsilon_{J j}-v^{k} \beta_{J k j}\right)\right\}
\end{aligned}
$$

All the non-relativistic transport coefficients are fixed in terms of relativistic transport coefficients and other thermodynamic variables. Explicit relations have been provided in the main text.

- In charge current we identify the thermal Hall contribution $\bar{\kappa}_{I} \epsilon^{i j} \nabla_{j} \tau$ (where $\tau$ is the temperature of non-relativistic fluid). The Hall energy flow sourced by the temperature gradient is known as the Leduc-Righi effect. It has been predicted in condensed

\footnotetext{
${ }^{3}$ We started out with a relativistic fluid in Landau frame, due to which Light Cone Reduction gives a non-relativistic fluid also in a particular frame. A trivial transformation of fluid velocity parameter $v^{i} \rightarrow v^{i}+\alpha(\mu, T) \epsilon^{i j} v_{j}$, can give many other terms out of which Hall viscosity will also be one. Since we reduce the fluid in a particular hydrodynamic frame, those terms do not appear.
} 
matter physics, that this kind of effect can be observed in various topological insulators.

- We also obtain the electromagnetic Hall energy current (parity-odd) $\bar{\sigma}_{I J} \epsilon^{i j}\left(\epsilon_{J j}-v^{k} \beta_{J k j}\right)$, where $\epsilon_{J j}$ is the applied electric field and $\beta_{I i j}$ is proportional to the applied magnetic field (check eq. (3.7) for exact definitions).

- We find that the non-relativistic fluid system satisfies the famous 'Wiedemann-Franz Law' for single component fluid.

- Finally, we compute the entropy current $j_{S}^{i}$ for the non-relativistic fluid in section 5 , and demand that the rate of entropy production of the system is always positive definite. For parity-even fluid it turns out to be quite straight forward. The canonical form of the entropy current confirms that the total entropy always increases due to dissipation, thermal and charge conduction. However for parity-odd fluid, canonical form of the entropy current fails to confirm the second law of thermodynamics. Therefore, we need to add two parity-odd vectors to the entropy current with arbitrary coefficients. Upon demanding the validity of second law, we see that one can fix these two coefficients exactly.

- Finally we observe that, unless the fluid is incompressible, all the parity-odd transport coefficients must vanish to satisfy the second law of thermodynamics.

\section{Relativistic anomalous charged fluid}

Hydrodynamic description does not follow from the action principle, rather it is generally formulated in the language of equations of motion. The reason for this is the presence of dissipation in thermal media. Due to internal friction called viscosity, a dissipative fluid loses its energy over time as it propagates. The fluid without any viscous drag is called an ideal fluid. In the simplest case, the hydrodynamic equations (for a uncharged fluid) are the laws of conservation of energy and momentum,

$$
\nabla_{\mu} T^{\mu \nu}=0 .
$$

A relativistic fluid in $(d+1,1)$ dimensions $(d+1$ spatial and 1 time dimensions $)$ has total $d+3$ independent intensive fluid variables: temperature $(T)$, pressure $(P)$ and velocities $u^{\mu}$ (only $d+1$ components of the velocity are independent due to normalization $u^{\mu} u_{\mu}=$ $-1)$. Densities of extensive variables: energy density $(E)$ and entropy density $(S)$ are not considered to be independent as they can be determined in terms of the intensive variables, by the first law of thermodynamics and the equation of state.

Additionally we have an Euler's relation which follows from the extensivity of the internal energy:

$$
E+P=T S,
$$

which in conjunction with the first law relates $P$ and $T$ as:

$$
\mathrm{d} P=S \mathrm{~d} T .
$$


Therefore, among these thermodynamic variables, we can consider only one to be independent, which we choose to be $T$. Thus, fluid is determined in terms of $d+2$ variables: $u^{\mu}, T$. On the other hand we have $d+2$ constitutive equations (2.1), and hence fluid system is completely determined. We can express the energy momentum tensor $T^{\mu \nu}$ as a function of temperature, velocity and their derivatives.

In case of fluid with multiple conserved $\mathrm{U}(1)$ currents, we have more fluid variables: charge densities $\left(Q_{I}\right)$. The corresponding conjugate variables are called chemical potentials denoted by $\left(M_{I}\right)$. In presence of conserved charges the first law is modified to

$$
\mathrm{d} E=T \mathrm{~d} S+M_{I} \mathrm{~d} Q_{I}
$$

From this equation one can compute the chemical potentials of the fluid in terms of charges and other variables. However, in our computation we consider chemical potentials $M_{I}$ to be our independent variables, not the charges. As a result, number of fluid variables have been increased to $d+3$. However, in this case we also have another constitutive equation (which follows from the global U(1) symmetries),

$$
\nabla_{\mu} J_{I}^{\mu}=0
$$

Euler's relations are also modified to:

$$
E+P=T S+M_{I} Q_{I}
$$

and from the first law one can write,

$$
\mathrm{d} P=S \mathrm{~d} T+Q_{I} \mathrm{~d} M_{I}
$$

Therefore, for charged fluid we consider our basic thermodynamic variables to be $T$ and $M_{I} / T$, rest are fixed in terms of these two variables. We can therefore write the last equation in the following form:

$$
\mathrm{d} P=\frac{E+P}{T} \mathrm{~d} T+T Q_{I} \mathrm{~d}\left(\frac{M_{I}}{T}\right)
$$

Thus, for a charged fluid, we express energy-momentum tensor and charge current as a function of fluid velocities, temperature, chemical potential and their derivatives.

One striking feature of a relativistic quantum field theory is triangle anomalies. The effect of this anomaly is reflected in three point correlation function (loop level) of charge currents. However, the anomaly does not affect the conservation of the current associated with a global symmetry in absence of external electromagnetic fields. When we put the theory in external background gauge fields coupled to the currents, some of the currents will no longer be conserved.

In the next subsection we discuss about the form of the energy momentum tensor and global U(1) current of a relativistic anomalous fluid in presence of background gauge fields. 


\subsection{Anomalous charged fluid: energy-momentum tensor and current}

We review a generic charged relativistic fluid in $(d+2)$-dim with anomalies. The energymomentum tensor is given by:

$$
T^{\mu \nu}=(E+P) u^{\mu} u^{\nu}+P g^{\mu \nu}+\Pi^{\mu \nu},
$$

where,

$$
\Pi^{\mu \nu}=-2 \eta \tau^{\mu \nu}-\zeta \theta^{\mu \nu},
$$

and fluid velocities are normalized

$$
u^{\mu} u_{\mu}=-1 .
$$

Here, $\eta$ and $\zeta$ are relativistic shear and bulk viscosity coefficients respectively. Up to first order in derivatives, the respective terms are given by:

$$
\begin{aligned}
& \tau^{\mu \nu}=\frac{1}{2} P^{\mu \alpha} P^{\nu \beta}\left[\nabla_{\alpha} u_{\beta}+\nabla_{\beta} u_{\alpha}-\frac{2}{d+1} g_{\alpha \beta} \theta\right], \\
& \theta^{\mu \nu}=\theta P^{\mu \nu}, \quad \theta=\nabla_{\alpha} u^{\alpha},
\end{aligned}
$$

where we use the projection operator:

$$
P^{\mu \nu}=g^{\mu \nu}+u^{\mu} u^{\nu}
$$

Similarly charge current of a relativistic fluid in presence of multiple charges $Q_{I}(I=$ $1,2,3)$ is given $b^{4}$

$$
J_{I}^{\mu}=Q_{I} u^{\mu}+\Upsilon_{I}^{\mu},
$$

where,

$$
\begin{aligned}
\Upsilon_{I}^{\mu} & =-\varrho_{I J} P^{\mu \nu} \nabla_{\nu}\left(\frac{M_{J}}{T}\right)+\lambda_{I J} E_{J}^{\mu}-\gamma_{I} P^{\mu \nu} \nabla_{\nu} T+\left\{\mho_{I} l^{\mu}+\tilde{\mho}_{I J} B_{J}^{\mu}\right\} \\
l^{\mu} & =\epsilon^{\mu \alpha \beta \gamma} u_{\alpha} \nabla_{\beta} u_{\gamma} .
\end{aligned}
$$

Here $\varrho_{I J}, \lambda_{I J}$ and $\gamma_{I}$ are charge, electric and thermal ${ }^{5}$ conductivities respectively. However, demanding the positivity of local entropy current one can show show that, $\gamma_{I}$ vanishes and $\varrho_{I J}$ is related to $\lambda_{I J}$.

We have kept the fluid in some background electromagnetic gauge fields given by $A_{I}^{\mu}$ :

$$
\begin{aligned}
F_{I}^{\mu \nu} & =\nabla^{\mu} A_{I}^{\nu}-\nabla^{\nu} A_{I}^{\mu}, \\
E_{I}^{\mu} & =F_{I}^{\mu \nu} u_{\nu}, \quad B_{I}^{\mu}=\frac{1}{2} \epsilon^{\mu \nu \alpha \beta} u_{\nu} F_{I \alpha \beta} .
\end{aligned}
$$

The last two terms of eq. (2.16) are the most generic single-derivative parity-odd modifications to the charge current allowed in Landau frame. These terms are specific to

\footnotetext{
${ }^{4}$ See [17] for a beautiful review.

${ }^{5}$ Thermal conductivity of relativistic theory is fundamentally different from the thermal conductivity of non-relativistic theory. Entropy positivity demands relativistic thermal conductivity to vanish, however in non-relativistic limit it origins from elsewhere.
} 
$(3+1)$-dimensions. In higher dimensions, the parity odd terms appear only at higher derivative orders. However, we would like to keep our calculations generic to $(d+2)$-dim in parity-even sector while still keeping the parity-odd sector specific to $d=2$. Therefore in all expressions, we represent parity-odd terms in curly braces $\{\ldots\}$, with an understanding that these terms contribute only for $d=2$.

We are working in Landau frame, which demands

$$
u_{\mu} \Pi^{\mu \nu}=0, \quad u_{\mu} \Upsilon_{I}^{\mu}=0
$$

and hence:

$$
u_{\mu} T^{\mu \nu}=-E u^{\nu}, \quad u_{\mu} J_{I}^{\mu}=-Q_{I} .
$$

The constitutive equations for this fluid are given by:

$$
\begin{aligned}
\nabla_{\mu} T^{\mu \nu} & =F_{I}^{\nu \lambda} J_{I \lambda}, \\
\nabla_{\mu} J_{I}^{\mu} & =\left\{C_{I J K} E_{J}^{\mu} B_{K \mu}\right\} .
\end{aligned}
$$

$C_{I J K}$ is called anomaly coefficient, which is completely symmetric in all indices.

Leading order $T^{\mu \nu}$ and $J^{\mu}$ must obey the first order conservation equations, which can be found trivially using eq. (2.22), (2.23):

$$
\begin{aligned}
u^{\alpha} \nabla_{\alpha} E & =-(E+P) \theta, \\
P^{\mu \alpha} \nabla_{\alpha} P-Q_{I} E_{I}^{\mu} & =-(E+P) u^{\alpha} \nabla_{\alpha} u^{\mu}, \\
u^{\mu} \nabla_{\mu} Q_{I}+Q_{I} \theta & =\left\{C_{I J K} E_{J}^{\mu} B_{K \mu}\right\} .
\end{aligned}
$$

We have projected the equations along and perpendicular to the direction of velocity for later convenience. We have also used $E_{I}^{\mu} u_{\mu}=0$, which can be seen trivially. Using these, $\tau^{\mu \nu}$ from eq. (2.12) can be written as:

$$
\tau^{\mu \nu}=\frac{1}{2}\left[\mathbf{Y}^{\mu \nu}+\mathbf{Y}^{\nu \mu}-\frac{2}{d+1} g^{\mu \nu} \theta-\mathbf{Z} u^{\mu} u^{\nu}\right],
$$

where,

$$
\begin{aligned}
\mathbf{Z} & =\frac{2}{d+1} \frac{u^{\alpha} \nabla_{\alpha} \mathbf{T}}{(E+P)}, \\
\mathbf{T} & =(d+1) P-E, \\
\mathbf{Y}^{\mu \nu} & =\nabla^{\mu} u^{\nu}-\frac{u^{\nu} \nabla^{\mu} P}{E+P}+Q_{I} \frac{u^{\nu} E_{I}^{\mu}}{E+P} .
\end{aligned}
$$

The trace of the energy-momentum tensor is given by:

$$
T_{\mu}^{\mu}=\mathbf{T}-\zeta(d+1) \theta .
$$

For conformal fluids $T_{\mu}^{\mu}=0$, which can be reached by setting $\mathbf{T}=\zeta=0$. 
Later, we shall use some relations involving $\mathbf{Y}^{\mu \nu}$, which can be found trivially using eq. (2.30):

$$
\begin{aligned}
& u_{\mu} \mathbf{Y}^{\mu \nu}=-\frac{2 u^{\nu} u^{\mu} \nabla_{\mu} P}{E+P}-\frac{\nabla^{\nu} P}{E+P}+\frac{Q_{I} E_{I}^{\nu}}{E+P}, \\
& u_{\nu} \mathbf{Y}^{\mu \nu}=\frac{\nabla^{\mu} P}{E+P}-\frac{Q_{I} E_{I}^{\mu}}{E+P} .
\end{aligned}
$$

\subsection{Entropy current}

In this sub-section we write down the expression for entropy current of a relativistic charged anomalous fluid and derive constraints on various transport coefficients which follow from second law of thermodynamics. The canonical form of relativistic entropy current is given by

$$
J_{S}^{\mu}=S u^{\mu}-\frac{M_{I}}{T} \Upsilon_{I}^{\mu}
$$

Using conservation equations eq. (2.22), (2.23) and thermodynamics eq. (2.6), (2.7) we can write:

$$
\nabla_{\mu} J_{S}^{\mu}=-\frac{1}{T} \Pi^{\mu \nu} \nabla_{\mu} u_{\nu}+\left[\frac{E_{I \mu}}{T}-\nabla_{\mu}\left(\frac{M_{I}}{T}\right)\right] \Upsilon_{I}^{\mu}
$$

Using eq. (2.10), (2.16) it can be written (up to second order in derivative) as

$$
\begin{aligned}
\nabla_{\mu} J_{S}^{\mu}= & \frac{1}{T} \eta \tau^{\mu \nu} \tau_{\mu \nu}+\frac{1}{T} \zeta \theta^{2}+\frac{1}{T} E_{I \mu} \lambda_{I J} E_{J}^{\mu}+\left[P_{\alpha \mu} \nabla^{\mu}\left(\frac{M_{I}}{T}\right)\right] \varrho_{I J}\left[P^{\alpha \nu} \nabla_{\nu}\left(\frac{M_{J}}{T}\right)\right] \\
& -\frac{1}{T} E_{I}^{\mu}\left(\varrho_{I J}+T \lambda_{J I}\right) \nabla_{\mu}\left(\frac{M_{J}}{T}\right)-\frac{1}{T} \gamma_{I} E_{I}^{\mu} \nabla_{\mu} T+\nabla_{\mu}\left(\frac{M_{I}}{T}\right) \gamma_{I} P^{\mu \nu} \nabla_{\nu} T \\
& +\left\{-C_{K I J} \frac{M_{K}}{T} E_{I \mu} B_{J}^{\mu}+\left(\mho_{I} l^{\mu}+\tilde{\mho}_{I J} B_{J}^{\mu}\right)\left[\frac{1}{T} E_{I \mu}-\nabla_{\mu}\left(\frac{M_{I}}{T}\right)\right]\right\} .
\end{aligned}
$$

Demanding entropy positivity, $\nabla_{\mu} J_{S}^{\mu} \geq 0$ we will get

$$
\gamma_{I}=0, \quad \eta \geq 0, \quad \zeta \geq 0, \quad \lambda_{I J}=\frac{1}{T} \varrho_{I J}
$$

$\varrho_{I J}$ matrix is positive definite.

However the last two terms of eq. (2.36) cannot be made positive definite. One would then expect that coefficients of those terms must vanish! But in [4], the authors identified that eqn (2.35) is not the most generic expression for the entropy current. They modified the entropy current with the most generic parity odd vectors allowed by the symmetry

$$
J_{S}^{\mu} \rightarrow J_{S}^{\mu}+\left\{D l^{\mu}+\tilde{D}_{I} B_{I}^{\mu}\right\}
$$


In presence of these two terms the parity-odd part of the last equation becomes,

$$
\begin{aligned}
& \left\{\nabla_{\mu}\left(D l^{\mu}+\tilde{D}_{I} B_{I}^{\mu}\right)-C_{K I J} \frac{M_{K}}{T} E_{I \mu} B_{J}^{\mu}+\left(\mho_{I} l^{\mu}+\tilde{\mho}_{I J} B_{J}^{\mu}\right)\left[\frac{1}{T} E_{I \mu}-\nabla_{\mu}\left(\frac{M_{I}}{T}\right)\right]\right\} \\
= & \left\{l^{\mu}\left[\nabla_{\mu} D-\frac{2 D}{E+P} \nabla_{\mu} P-\mho_{I} \nabla_{\mu}\left(\frac{M_{I}}{T}\right)\right]+l^{\mu} E_{I \mu}\left[D \frac{2 Q_{I}}{E+P}-2 \tilde{D}_{I}+\frac{1}{T} \mho_{I}\right]\right. \\
& +B_{J}^{\mu}\left[\nabla_{\mu} \tilde{D}_{J}-\frac{\tilde{D}_{J}}{E+P} \nabla_{\mu} P-\tilde{\mho}_{I J} \nabla_{\mu}\left(\frac{M_{I}}{T}\right)\right] \\
& \left.+E_{I}^{\mu} B_{J \mu}\left[\tilde{D}_{J} \frac{Q_{I}}{E+P}+\frac{1}{T} \tilde{\mho}_{I J}-C_{K I J} \frac{M_{K}}{T}\right]\right\} \\
= & 0 .
\end{aligned}
$$

Demanding the positivity of divergence of local entropy current we get,

$$
\begin{aligned}
\frac{\partial D}{\partial P} & =\frac{2 D}{E+P}, \quad \frac{\partial D}{\partial\left(M_{I} / T\right)}=\mho_{I}, \\
\frac{\partial \tilde{D}_{J}}{\partial P} & =\frac{\tilde{D}_{J}}{E+P}, \quad \frac{\partial \tilde{D}_{J}}{\partial\left(M_{I} / T\right)}=\tilde{\mho}_{I J}, \\
D \frac{2 Q_{I}}{E+P}-2 \tilde{D}_{I} & =-\frac{1}{T} \mho_{I}, \\
\tilde{D}_{J} \frac{Q_{I}}{E+P} & =-\frac{1}{T} \tilde{\mho}_{I J}+C_{K I J} \frac{M_{K}}{T} .
\end{aligned}
$$

One can in principle solve the above set of equation to get relations between $D, \tilde{D}_{I}, \mho_{I}, \tilde{\mho}_{I J}$ and $C_{I J K}$.

Finally we have the desired form of entropy current

$\nabla_{\mu} J_{S}^{\mu}=\frac{1}{T} \eta \tau^{\mu \nu} \tau_{\mu \nu}+\frac{1}{T} \zeta \theta^{2}+\left[\frac{E_{I}^{\alpha}}{T}-P^{\alpha \mu} \nabla_{\mu}\left(\frac{M_{I}}{T}\right)\right] \varrho_{I J}\left[\frac{E_{J \alpha}}{T}-P_{\alpha \nu} \nabla^{\nu}\left(\frac{M_{J}}{T}\right)\right]$

which is entirely positive definite.

\section{Non-relativistic charged fluid}

In this section, we review the properties of charged non-relativistic dissipative fluid living in $(d+1)$-dimensions, in presence of some background gauge fields. A non-relativistic fluid has following constitutive equations:

Continuity equation:

$$
\partial_{t} \rho+\partial_{i}\left(\rho v^{i}\right)=0
$$

where $\rho$ is density of fluid particle.

Momentum conservation equation:

$$
\partial_{t}\left(\rho v^{j}\right)+\partial_{i}\left(t^{i j}\right)=0
$$

where $t^{i j}$ is the energy-momentum tensor. 
Energy conservation equation:

$$
\partial_{t}\left(\epsilon+\frac{1}{2} \rho \mathbf{v}^{2}\right)+\partial_{i} j^{i}=0
$$

where $j^{i}$ is the energy current.

Conservation of charge current:

$$
\partial_{t} q_{I}+\partial_{i} j_{I}^{i}=0
$$

where, $q_{I}$ 's are the $\mathrm{U}(1)$ charge densities and $\vec{j}_{I}$ 's are the corresponding currents. Note that energy current is denoted by $\vec{j}$ where as charge current is denoted by $\vec{j}_{I}$.

In $d$ spatial dimensions, charged fluid has total $d+3$ independent variables (pressure $p$, temperature $\tau$, chemical potentials $\mu_{I}$ and velocity $\vec{v}$ ), while others (density $\rho$, energy density $\epsilon$, charge density $q_{I}$ and entropy density $s$ ) are fixed in terms of these using thermodynamic relations. Unlike the relativistic case we do not consider the Euler's relation here. Later we shall see that the light-cone reduction does not preserve extensivity of the fluid system. Hence, the fluid is determined by $d+3$ parameters chosen to be: $\tau, \mu_{I} / \tau, p$ and $\vec{v}$ (same as $(\mathrm{d}+1,1)$-dim relativistic fluid). On the other hand we have $d+3$ constitutive equations.

Therefore, we can express energy-momentum tensor and charge current in terms of $\tau$, $\mu_{I} / \tau, p, \vec{v}$ and their derivatives. For ideal fluid they are given by,

$$
\begin{aligned}
t^{i j} & =\rho v^{i} v^{j}+p g^{i j}, \\
j^{i} & =\left(\epsilon+p+\frac{1}{2} \rho \mathbf{v}^{2}\right) v^{i}, \\
j_{I}^{i} & =q_{I} v^{i} .
\end{aligned}
$$

\subsection{Non-relativistic dissipative fluid in background electromagnetic field}

When we put a dissipative fluid in background electromagnetic fields, the above equations are modified. Continuity equation and charge conservation equation remain the same but other two equations are modified to

$$
\begin{aligned}
\partial_{t}\left(\rho v^{j}\right)+\partial_{i}\left(t^{i j}\right) & =q_{I} \epsilon_{I}^{j}-j_{I i} \beta_{I}^{i j}, \\
\partial_{t}\left(\epsilon+\frac{1}{2} \rho \mathbf{v}^{2}\right)+\partial_{i} j^{i} & =j_{I}^{i} \epsilon_{I i},
\end{aligned}
$$

where,

$$
\epsilon_{I}^{i}=-\partial^{i} \phi_{I}-\partial_{t} a_{I}^{i}, \quad \beta_{I}^{i j}=\partial^{i} a_{I}^{j}-\partial^{j} a_{I}^{i},
$$

are electric and magnetic fields respectively. $\phi_{I}$ and $a_{I}^{i}$ are scalar and vector potentials respectively.

For dissipative fluid the stress-energy tensor is given by,

$$
t^{i j}=\rho v^{i} v^{j}+p g^{i j}+\pi^{i j}
$$


where $\pi^{i j}$ is the correction to ideal fluid stress tensor due to dissipation

$$
\begin{aligned}
\pi^{i j} & =-n \sigma^{i j}-z \delta^{i j} \partial_{k} v^{k}, \\
\sigma^{i j} & =\partial^{i} v^{j}+\partial^{j} v^{i}-\delta^{i j} \frac{2}{d} \partial_{k} v^{k} .
\end{aligned}
$$

$n$ and $z$ are the non-relativistic shear and bulk viscosity coefficients respectively.

The energy current for dissipative fluid is given by,

$$
j^{i}=\left(\epsilon+p+\frac{1}{2} \rho \mathbf{v}^{2}\right) v^{i}+\varsigma^{i}
$$

where,

$$
\varsigma^{i}=-n \sigma^{i j} v^{j}-z \partial_{k} v^{k} v^{i}-\kappa \partial^{i} \tau-\xi_{I} \nabla^{i}\left(\frac{\mu_{I}}{\tau}\right)+\sigma_{I} \epsilon_{I}^{i}-\alpha_{I} v_{j} \beta_{I}^{j i} .
$$

Here $\kappa, \xi_{I}, \sigma_{I}$ and $\alpha_{I}$ are thermal, charge, electric and magnetic conductivities respectively. Later we shall see that, for non-relativistic fluid, obtained by light cone reduction of a relativistic fluid, $\xi_{I}$ and $\alpha_{I}$ are related to $\sigma_{I}$.

Similarly charge current for a dissipative fluid is given by,

$$
j_{I}^{i}=q_{I} v^{i}+\varsigma_{I}^{i},
$$

where,

$$
\begin{aligned}
\varsigma_{I}^{i}= & -\tilde{\kappa}_{I} \nabla^{i} \tau-\tilde{\xi}_{I J} \nabla^{i}\left(\frac{\mu_{J}}{\tau}\right)-\tilde{m}_{I} \nabla^{i} p+\tilde{\sigma}_{I J} \epsilon_{J}^{i} \\
& -\tilde{\alpha}_{I J} v_{k} \beta_{J}^{k i}+\left\{\bar{\kappa}_{I} \epsilon^{i j} \nabla_{j} \tau+\bar{\xi}_{I J} \epsilon^{i j} \nabla_{j}\left(\frac{\mu_{J}}{\tau}\right)-\bar{m}_{I} \epsilon^{i j} \nabla_{j} p+\bar{\sigma}_{I J} \epsilon^{i j} \epsilon_{J j}-\bar{\alpha}_{I J} \epsilon^{i j} v^{k} \beta_{J k j}\right\} .
\end{aligned}
$$

$\tilde{\kappa}_{I}, \tilde{\sigma}_{I J}, \tilde{\xi}_{I J}, \tilde{m}_{I}, \tilde{\alpha}_{I J}$ and $\bar{\kappa}_{I}, \bar{\sigma}_{I J}, \bar{\xi}_{I J}, \bar{m}_{I}, \bar{\alpha}_{I J}$ are some arbitrary parity-even and parity-odd transport coefficients. ${ }^{6}$ Again, for a non-relativistic fluid, obtained by light-cone reduction these transport coefficients are fixed in terms of relativistic transport coefficients. Also, they are constrained when we demand positivity of local entropy current.

\section{Light-cone reduction}

Discrete light cone quantization of a $(d+1,1)$-dim relativistic quantum field theory (QFT) boils down to a non-relativistic quantum field theory in one lower dimension. Let us consider a QFT in flat spacetime with metric,

$$
d s^{2}=-\left(d x^{0}\right)^{2}+\left(d x^{d+1}\right)^{2}+\sum_{i=1}^{d}\left(d x^{i}\right)^{2} .
$$

We introduce the light-cone coordinates,

$$
x^{ \pm}=\frac{1}{\sqrt{2}}\left(x^{0} \pm x^{d+1}\right) .
$$

\footnotetext{
${ }^{6}$ The sign of these coefficients are completely arbitrary for now, and are chosen keeping in mind later convenience.
} 
In the light-cone frame the metric can be written as,

$$
d s^{2}=-2 d x^{+} d x^{-}+\sum_{i=1}^{d}\left(d x^{i}\right)^{2} .
$$

Suppose we view the QFT in this light-cone frame and evolve it in light-cone time $x^{+}$. Then, for fixed light-cone momentum $P_{-}$, we obtain a system in $d+1$ dimensions with non-relativistic invariance. This is because the symmetry algebra of the relativistic theory reduces to corresponding non-relativistic symmetry algebra upon light-cone reduction. For example, if the relativistic theory is invariant under Poincaré transformation, then the corresponding algebra $(\mathrm{SO}(\mathrm{d}+1,1))$ reduces to $d$ dimensional Galilean algebra. On the other hand if we consider a QFT with conformal invariance in $(d+1)+1$ dimensions, then upon light cone reduction the corresponding algebra $(\mathrm{SO}(\mathrm{d}+2,2))$ reduces to Schrödinger algebra in $d$ spatial dimensions. This is known as discrete light-cone quantization of quantum field theories.

Since hydrodynamics is low energy fluctuation of equilibrium quantum field theory, light-cone reduction of relativistic constitutive equations boil down to the non-relativistic constitutive equations for a fluid in one lower dimension. Relativistic charged fluid in $(d+1,1)$-dim, as we have already discussed, has $d+3$ degrees of freedom: temperature, chemical potential and normalized velocities. On the other had, a non-relativistic fluid in $d$ spatial dimensions also has total $d+3$ degrees of freedom: temperature, pressure, chemical potential and velocities. Our goal is to consider the most generic relativistic anomalous fluid system in presence of background electromagnetic fields in $(3+1)$ dimensions and reduce the constitutive equation (also equation for entropy current) over light-cone coordinates and obtain the corresponding non-relativistic equations for a fluid in one lower dimensions. We also find a mapping between the degrees of freedom of the $(d+2)$-dimensional fluid to the degrees of freedom of the $(d+1)$-dimensional fluid.

We denote the $d$ spatial coordinates with $x^{i}$. Metric components in light-cone coordinates are given by

$$
g^{i j}=\delta^{i j} \quad \text { and } \quad g^{+-}=-1,
$$

rest are zero. Gradient operator is given by,

$$
\nabla_{\mu}=\left\{\partial_{+}, \partial_{-}, \partial_{i}\right\} \quad \text { and } \quad \nabla^{\mu}=\left\{-\partial_{-},-\partial_{+}, \partial_{i}\right\}
$$

As we have seen that not all components of $u^{\mu}, J_{I}^{\mu}$ and $T^{\mu \nu}$ are independent, from eq. (2.21) we get,

$$
\begin{aligned}
u^{-} & =\frac{1}{2 u^{+}}\left(1+\mathbf{u}^{2}\right), \\
u^{+} J_{I}^{-} & =Q_{I}+u_{i} J_{I}^{i}-u^{-} J_{I}^{+}, \\
u^{+} T^{\mu-} & =E u^{\mu}+u_{i} T^{\mu i}-u^{-} T^{\mu+} .
\end{aligned}
$$

We shall reduce the theory along the $x^{-}$direction, and consider $x^{+}$to be the non-relativistic time. We will consider only solutions to the relativistic equations that do not depend on $x^{-}$; that is, all derivatives $\partial_{-}$vanish. 


\subsection{Reduction of background fields}

We reduce the Maxwell's equations for the background fields of relativistic fluid to get consistent background for non-relativistic theory. Maxwell's equations for relativistic system are given by (we shall assume that the sources for these background fields are far away from the fluid),

$$
\nabla_{\mu} F_{I}^{\mu \nu}=0
$$

Under light-cone reduction the above equations take the following form:

$$
\begin{aligned}
\vec{\nabla}^{2} A_{I}^{+} & =0, \\
\nabla_{i}\left(\nabla^{i} A_{I}^{-}+\nabla_{+} A_{I}^{i}\right) & =-\nabla_{+}^{2} A_{I}^{+}, \\
\nabla_{i}\left(\nabla^{i} A_{I}^{j}-\nabla^{j} A_{I}^{i}\right) & =\nabla^{j} \nabla_{+} A_{I}^{+} .
\end{aligned}
$$

These equations can be identified with source free static Maxwell's equations of a nonrelativistic system if we map: ${ }^{7}$

$$
\begin{aligned}
A_{I}^{-} & =\phi_{I} \quad(\text { scalar potential }) \\
A_{I}^{i} & =a_{I}^{i} \quad(\text { vector potential })
\end{aligned}
$$

and

$$
\nabla^{i} \nabla_{i} A_{I}^{+}=0, \quad \nabla_{+} \nabla_{+} A_{I}^{+}=0, \quad \nabla^{i} \nabla_{+} A_{I}^{+}=0 .
$$

Which would inturn tell us

$$
\nabla_{+} A_{I}^{+}, \nabla_{i} A_{I}^{+}=\text {constant. }
$$

We would however like $A_{I}^{+}$to have some finite value at infinity, which will enforce:

$$
A_{I}^{+}=\text {constant }
$$

With this identification, from eq. (2.19) one can show that

$$
E_{I}^{+}=0, \quad E_{I}^{-}=u_{i} \epsilon_{I}^{i}, \quad E_{I}^{i}=u^{+} \epsilon_{I}^{i}-u_{j} \beta_{I}^{j i},
$$

and

$$
\begin{aligned}
B_{I}^{+} & =-u^{+} \epsilon_{i j} \beta_{I}^{i j}, \quad B_{I}^{-}=u^{-} \epsilon_{i j} \beta_{I}^{i j}-2 \epsilon_{i j} u^{i} \epsilon_{I}^{j}, \\
B_{I}^{i} & =-2 u^{+} \epsilon_{i j} \epsilon_{I}^{j},
\end{aligned}
$$

where, $\epsilon_{I}^{i}=-\partial^{i} \phi_{I}-\partial_{t} a_{I}^{i}$ and $\beta_{I}^{i j}=\partial^{i} a_{I}^{j}-\partial^{j} a_{I}^{i}$.

\footnotetext{
${ }^{7}$ In appendix A we have discussed how to expand Maxwell's equations in powers of $1 / c$ to get the non-relativistic limit.
} 
Finally let us check what happens to the gauge freedom in relativistic side. The gauge transformation is give by,

$$
A^{\mu} \rightarrow A^{\mu}+\nabla^{\mu} \Lambda
$$

In the light-cone coordinates it becomes

$$
\begin{aligned}
& A^{+} \rightarrow A^{+}, \\
& A^{-} \rightarrow A^{-}-\nabla_{+} \Lambda, \\
& A^{i} \rightarrow A^{i}+\nabla^{i} \Lambda .
\end{aligned}
$$

Thus we see that the gauge freedom of relativistic theory reduces to the gauge freedom of the non-relativistic theory. Additionally $A^{+}$, which we have fixed to be a constant, under a gauge choice does not change. Otherwise, one could perform a local gauge transformation to modify $A^{+}$to a non constant value, which would then break our identification.

\subsection{Reduction of energy-momentum tensor and charge current}

The reduction of relativistic equations of energy-momentum and charge conservation after using eq. (4.14) are given by:

$$
\begin{aligned}
\nabla_{+} T^{++}+\nabla_{i} T^{i+} & =0 \\
\nabla_{+} T^{+-}+\nabla_{i} T^{i-} & =-J_{I}^{i}\left(\nabla_{i} A_{I}^{-}+\nabla_{+} A_{I i}\right), \\
\nabla_{+} T^{+j}+\nabla_{i} T^{i j} & =-J_{I}^{+}\left(\nabla_{+} A_{I}^{j}+\nabla^{j} A_{I}^{-}\right)-J_{I}^{i}\left(\nabla_{i} A_{I}^{j}-\nabla^{j} A_{I i}\right), \\
\nabla_{+} J_{I}^{+}+\nabla_{i} J_{I}^{i} & =\left\{C_{I J K} E_{J}^{\mu} B_{K \mu}\right\}=0 .
\end{aligned}
$$

R.H.S. of eq. (4.22) can be shown to vanish explicitly after reduction in four dimensions.

These equations reduce to non-relativistic equations under following identifications

$$
\begin{aligned}
T^{++} & =\rho, & T^{i+} & =\rho v^{i}, \\
T^{+-} & =\epsilon+\frac{1}{2} \rho \mathbf{v}^{2}, & T^{i-} & =j^{i}, \\
J_{I}^{+} & =q_{I}, & J_{I}^{i} & =j_{I}^{i} .
\end{aligned} \quad T^{i j}=t^{i j},
$$

The identification also tells that the non-relativistic charge current is conserved, while the relativistic charge current was not. We shall now attempt to use this mapping to establish relations between relativistic and non-relativistic parameters.

A point worth noting is that identifications eq. (4.23) are not unique, to give the NR constitutive equations. NR fluid has only one vector constitutive equation, and LCR also gives just one vector equation, which will set sum of the identifications unique. However scalar equations from LCR can be mixed in any linear combination to give the scalar constitutive equations, to do which however one must introduce certain dimension-full constants to the theory. To avoid these constants we have avoided linear combinations and hence reached eq. (4.23). 


\subsection{1 $T^{++}$calculation}

Using eq. (2.27) and (2.13) we can show:

$$
\begin{aligned}
\tau^{++} & =-\frac{1}{2}\left(u^{+}\right)^{2} \mathbf{Z} \\
\theta^{++} & =\left(u^{+}\right)^{2} \theta
\end{aligned}
$$

and thus:

$$
T^{++}=(E+P)\left(u^{+}\right)^{2}+\left(u^{+}\right)^{2}(\eta \mathbf{Z}-\zeta \theta) .
$$

Identifying $T^{++}=\rho$

$$
\rho=(E+P)\left(u^{+}\right)^{2}+\left(u^{+}\right)^{2}(\eta \mathbf{Z}-\zeta \theta) .
$$

\subsection{2 $T^{i+}$ calculation}

Using eq. (2.27) and (2.13) we can show:

$$
\begin{aligned}
\tau^{i+} & =\frac{1}{2} \mathbf{Y}^{i}-\frac{1}{2} \mathbf{Z} u^{i} u^{+}, \\
\theta^{i+} & =u^{i} u^{+} \theta,
\end{aligned}
$$

where,

$$
\mathbf{Y}^{\mu}=\mathbf{Y}^{\mu+}=\left(\nabla^{\mu} u^{+}-\frac{u^{+} \nabla^{\mu} P}{E+P}+u^{+} \frac{Q_{I} E_{I}^{\mu}}{E+P}\right)
$$

Using eq. (4.26), we will find,

$$
T^{i+}=\frac{u^{i}}{u^{+}} \rho-\eta \mathbf{Y}^{i}
$$

Identifying $T^{i+}=\rho v^{i}$

$$
v^{i}=\frac{u^{i}}{u^{+}}-\frac{\eta}{\rho} \mathbf{Y}^{i}
$$

\subsection{3 $\quad T^{i j}$ calculation}

Using eq. (2.27) we will find:

$$
\tau^{i j}=\frac{1}{2}\left[\mathbf{Y}^{i j}+\mathbf{Y}^{j i}-\frac{2}{d+1} g^{i j} \theta-\mathbf{Z} u^{i} u^{j}\right] .
$$

It's easy to check that:

$$
-\frac{u^{\alpha} \nabla_{\alpha} E}{(E+P)}=\theta=-\frac{u^{\alpha} \nabla_{\alpha} P}{(E+P)}+u^{+} \nabla_{k} v^{k},
$$

and thus introducing $\mathbf{Z}$ from eq. (2.28),

$$
-\frac{2}{(d+1)} \theta=\frac{1}{d} \mathbf{Z}-u^{+} \frac{2}{d} \nabla_{k} v^{k} .
$$

Using the above relations and the identity:

$$
\mathbf{Y}^{\mu i}=u^{+} \nabla^{\mu} v^{i}+v^{i} \mathbf{Y}^{\mu}
$$


we find that:

$$
\tau^{i j}=\frac{1}{2}\left(u^{+} \sigma^{i j}+v^{i} \mathbf{Y}^{j}+v^{j} \mathbf{Y}^{i}+\frac{g^{i j}}{d} \mathbf{Z}-\left(u^{+}\right)^{2} v^{i} v^{j} \mathbf{Z}\right),
$$

where $\sigma^{i j}$ is given by eq. (3.10). Similarly using eq. (2.13) we can reach to:

$$
\theta^{i j}=-g^{i j} \frac{u^{\alpha} \nabla_{\alpha} P}{(E+P)}+g^{i j} u^{+} \nabla_{k} v^{k}+\left(u^{+}\right)^{2} v^{i} v^{j} \theta
$$

and thus:

$$
T^{i j}=v^{i} v^{j} \rho+g^{i j}\left[P-\left(\frac{\eta}{d} \mathbf{Z}-\zeta \frac{u^{\alpha} \nabla_{\alpha} P}{E+P}\right)\right]-\eta u^{+} \sigma^{i j}-\zeta u^{+} g^{i j} \nabla_{k} v^{k} .
$$

Identifying $T^{i j}=t^{i j}$

$$
\begin{aligned}
p & =P-\left(\frac{\eta}{d} \mathbf{Z}-\zeta \frac{u^{\alpha} \nabla_{\alpha} P}{E+P}\right), \\
n & =\eta u^{+} \\
z & =\zeta u^{+}
\end{aligned}
$$

\subsection{4 $T^{+-}$calculation}

Using eq. (4.8), (4.25) and (4.29) we can find:

$$
T^{+-}=\frac{1}{2}(E-P)-\frac{1}{2}(\eta \mathbf{Z}-\zeta \theta)+\frac{1}{2} \rho \mathbf{v}^{2} .
$$

Identifying $T^{+-}=\epsilon+\frac{1}{2} \rho \mathbf{v}^{2}$

$$
\epsilon=\frac{1}{2}(E-P)-\frac{1}{2}(\eta \mathbf{Z}-\zeta \theta)
$$

\subsection{5 $T^{i-}$ calculation}

Using eq. (4.8), (4.29) and (4.37) we can find:

$$
T^{i-}=v^{i}\left(\epsilon+p+\frac{1}{2} \rho \mathbf{v}^{2}\right)-n \sigma^{i k} v_{k}-z v^{i} \nabla_{k} v^{k}+\eta \frac{1}{\left(u^{+}\right)^{2}} \mathbf{Y}^{i} .
$$

The last term can be written as:

$$
\eta \frac{1}{\left(u^{+}\right)^{2}} \mathbf{Y}^{i}=-\eta \frac{1}{T} \nabla^{i}\left(\frac{T}{u^{+}}\right)-\eta u^{+} \frac{Q_{I} T}{\rho} \nabla^{i}\left(\frac{M_{I}}{T}\right)+\eta u^{+} \frac{Q_{I}}{\rho} E_{I}^{i} .
$$

We identify (leading order):

$$
\tau=\frac{T}{u^{+}}, \quad \mu_{I}=\frac{M_{I}}{u^{+}}, \quad q_{I}=Q_{I} u^{+},
$$

and

$$
\kappa=\frac{\eta}{\tau u^{+}}=2 n \frac{\epsilon+p}{\tau \rho}, \quad \sigma_{I}=n \frac{q_{I}}{\rho} .
$$


With this identification we get,

$$
\eta \frac{1}{\left(u^{+}\right)^{2}} \mathbf{Y}^{i}=-\kappa \nabla^{i} \tau-\tau \sigma_{I} \nabla^{i}\left(\frac{\mu_{I}}{\tau}\right)+\sigma_{I}\left(\epsilon_{I}^{i}-v_{j} \beta_{I}^{j i}\right) .
$$

Identifying $T^{i-}=j^{i}$ we have:

$j^{i}=v^{i}\left(\epsilon+p+\frac{1}{2} \rho \mathbf{v}^{2}\right)-n \sigma^{i k} v_{k}-z v^{i} \nabla_{k} v^{k}-\kappa \nabla^{i} \tau-\tau \sigma_{I} \nabla^{i}\left(\frac{\mu_{I}}{\tau}\right)+\sigma_{I}\left(\epsilon_{I}^{i}-v_{j} \beta_{I}^{j i}\right)$.

Comparing it with (3.12), we find that we have the expected form. Also we have estabilished relations between charge, electric and magnetic conductivities:

$$
\frac{\xi_{I}}{\tau}=\sigma_{I}=\alpha_{I} .
$$

\subsection{6 $J^{+}$calculation}

Using eq. (2.16) we can find

$$
\begin{aligned}
\Upsilon_{I}^{+} & =-\varrho_{I J} u^{+} u^{\nu} \nabla_{\nu}\left(\frac{M_{J}}{T}\right)-\gamma_{I} u^{+} u^{\nu} \nabla_{\nu} T+\left\{\mho_{I} l^{+}+\tilde{\mho}_{I J} B_{J}^{+}\right\}, \\
l^{+} & =-\epsilon^{+-i j}\left(u^{+}\right)^{2} \nabla_{i} v_{j}=-\epsilon^{i j}\left(u^{+}\right)^{2} \nabla_{i} v_{j} .
\end{aligned}
$$

Using eq. (4.16) we can write

$$
\Upsilon^{+}=-u^{+}\left[\varrho_{I J} u^{\nu} \nabla_{\nu}\left(\frac{M_{J}}{T}\right)+\gamma_{I} u^{\nu} \nabla_{\nu} T+\left\{\mho_{I} \epsilon^{i j} u^{+} \nabla_{i} v_{j}+\tilde{\mho}_{I J} \epsilon_{i j} \beta_{J}^{i j}\right\}\right] .
$$

Identifying $J_{I}^{+}=q_{I}$,

$$
q_{I}=u^{+} Q_{I}+u^{+} \varpi_{I}
$$

where,

$$
\varpi_{I}=-\left[\varrho_{I J} u^{\nu} \nabla_{\nu}\left(\frac{M_{J}}{T}\right)+\gamma_{I} u^{\nu} \nabla_{\nu} T+\left\{\mho_{I} \epsilon^{i j} u^{+} \nabla_{i} v_{j}+\tilde{\mho}_{I J} \epsilon_{i j} \beta_{J}^{i j}\right\}\right] .
$$

This tells us the sub-leading correction to non-relativistic charges.

\subsection{7 $J^{i}$ calculation}

Using eq. (2.16) we can find

$$
\Upsilon_{I}^{i}=-\varrho_{I J} P^{i \nu} \nabla_{\nu}\left(\frac{M_{J}}{T}\right)+\lambda_{I J} E_{J}^{i}-\gamma_{I} P^{i \nu} \nabla_{\nu} T+\left\{\mho_{I} l^{i}+\tilde{\mho}_{I J} B_{J}^{i}\right\} .
$$

One can find using eq. (4.44) that:

$$
\left\{l^{i}\right\}=\left\{v^{i} l^{+}+\left(u^{+}\right)^{2} \Xi^{i}\right\},
$$

where

$$
\left\{\Xi^{i}\right\}=\left\{\epsilon^{i j}\left(\frac{1}{\tau\left(u^{+}\right)^{2}} \nabla_{j} \tau+\frac{q_{I} t}{\rho} \nabla_{j}\left(\frac{\mu_{I}}{\tau}\right)-\frac{2}{\rho} \nabla_{j} p+\frac{1}{\rho} Q_{J} E_{J j}\right)\right\} .
$$


Certain velocity dependent terms appear in the above expression, which can be shown to sum to zero in 2 spatial dimensions.

Using thermodynamic relations eq. (2.6), (2.7), (4.44), along with all light cone identifications, we will find

$$
\begin{aligned}
J_{I}^{i}= & v^{i} J_{I}^{+}-\frac{\gamma_{I} u^{+} \tau}{2(\epsilon+p)} \nabla^{i} p-\frac{\kappa q_{I}}{2(\epsilon+p)} \nabla^{i} \tau-\left(\varrho_{I J}+\xi_{I} \frac{q_{J}}{2(\epsilon+p)}-\gamma_{I} u^{+} \frac{\tau^{2} q_{J}}{2(\epsilon+p)}\right) \nabla^{i}\left(\frac{\mu_{J}}{\tau}\right) \\
& +\left(\frac{\sigma_{J} q_{I}}{2(\epsilon+p)}+\lambda_{I J} u^{+}\right)\left(\epsilon_{J}^{j}-v_{k} \beta_{J}^{k j}\right)-\left\{\mho_{I}\left(u^{+}\right)^{2} \frac{2}{\rho} \epsilon^{i j}\right\} \nabla_{j} p+\left\{\frac{\mho_{I}}{\tau} \epsilon^{i j}\right\} \nabla_{j} \tau \\
& +\left\{\mho_{I}\left(u^{+}\right)^{2} \frac{q_{J} \tau}{\rho} \epsilon^{i j}\right\} \nabla_{j}\left(\frac{\mu_{J}}{\tau}\right)+\left\{\mho_{I}\left(u^{+}\right)^{2} \frac{q_{J}}{\rho} \epsilon^{i j}-\tilde{\mho}_{I J} u^{+} 2 \epsilon^{i j}\right\}\left(\epsilon_{J j}-v^{k} \beta_{J k j}\right) .
\end{aligned}
$$

We identify

$$
\begin{array}{rlrl}
\tilde{m}_{I} & =\frac{\gamma_{I} \tau u^{+}}{2(\epsilon+p)}, & & \tilde{\xi}_{I J}=\left[\varrho_{I J}+\frac{\xi_{J} q_{I}}{2(\epsilon+p)}-m_{I} q_{J} \tau\right], \\
\tilde{\kappa}_{I}=\frac{\kappa q_{I}}{2(\epsilon+p)}, & \tilde{\sigma}_{I J}=\tilde{\alpha}_{I J}=\left[\lambda_{I J} u^{+}+\frac{\sigma_{J} q_{I}}{2(\epsilon+p)}\right], \\
\bar{m}_{I}=\frac{2 \omega_{I}}{\rho}, & \bar{\xi}_{I J}=\xi_{J} \frac{\omega_{I}}{n}, \\
\bar{\kappa}_{I}=\kappa \frac{\omega_{I}}{n}, & \bar{\sigma}_{I J}=\bar{\alpha}_{I J}=\left[\sigma_{J} \frac{\omega_{I}}{n}-2 \tilde{\omega}_{I J}\right],
\end{array}
$$

where,

$$
\omega_{I}=\mho_{I}\left(u^{+}\right)^{2}, \quad \tilde{\omega}_{I J}=\tilde{\mho}_{I J} u^{+} .
$$

Finally we have the desired form of charge current

$$
\begin{aligned}
j_{I}^{i}= & q_{I} v^{i}+\varsigma_{I}^{i}, \\
\varsigma_{I}^{i}= & -\tilde{\kappa}_{I} \nabla^{i} \tau-\tilde{\xi}_{I J} \nabla^{i}\left(\frac{\mu_{J}}{\tau}\right)-\tilde{m}_{I} \nabla^{i} p+\tilde{\sigma}_{I J}\left(\epsilon_{J}^{i}-v_{k} \beta_{J}^{k i}\right) \\
& +\left\{\bar{\kappa}_{I} \epsilon^{i j} \nabla_{j} \tau+\bar{\xi}_{I J} \epsilon^{i j} \nabla_{j}\left(\frac{\mu_{J}}{\tau}\right)-\bar{m}_{I} \epsilon^{i j} \nabla_{j} p+\bar{\sigma}_{I J} \epsilon^{i j}\left(\epsilon_{J j}-v^{k} \beta_{J k j}\right)\right\},
\end{aligned}
$$

Note that all transport coefficients are not independent. The only independent coefficients are: $\tilde{m}_{I}, \tilde{\xi}_{I J}, \tilde{\sigma}_{I J}, \omega_{I}$ and $\tilde{\omega}_{I J}$.

Wiedemann-Franz law. This famous law predicts that the ratio of charge conductivity (which appears in charge current) ${ }^{8}$ to thermal conductivity (which appears in energy current) in metals as: $\tilde{\sigma} / \kappa=1 / L \tau$, where $L$ is the Lorenz number predicted to be $\sim 2.45 \times 10^{-8} W \Omega K^{-2}$. The law is found to be in good agreement with experiments. We attempt to check the same in our setup: ${ }^{9}$

$$
\frac{\tilde{\sigma}}{\kappa}=\frac{\rho \varrho}{2 n(\epsilon+p)}+\frac{\tau q^{2}}{4(\epsilon+p)^{2}},
$$

\footnotetext{
${ }^{8}$ Lets consider only one U(1) charge here.

${ }^{9}$ We have used here the fact that non-relativistic system respects the constraint $\tau \tilde{\sigma}_{I J}=\tilde{\xi}_{I J}$, which has been showed in next section.
} 
We model the electrons in metals as free classical gas with no external pressure: fluid with homogeneous particles each of charge $e$ (electronic charge), mass $m_{e}$ and average energy $3 / 2 k_{B} \tau$. One can check that under mentioned assumptions, our system follows Wiedemann-Franz Law with Lorenz number given as:

$$
L=\left(\frac{\varrho}{n} \frac{m_{e}}{3 k_{B}}+\frac{e^{2}}{9 k_{B}^{2}}\right)^{-1},
$$

As we shall see later, for the case with only single kind of particles $(\rho \propto q)$, all the coefficients in our theory vanish except $n$. In this very particular case however, we can check that Lorenz number is given approximately by: $6.68 \times 10^{-8} W \Omega K^{-2}$, given our assumptions, which is in fair agreement with the experimental value.

\subsection{Thermodynamics of the reduced system}

We start with a relativistic system with variables satisfying the eq. (2.4) and (2.6). The first equation is the first law of thermodynamics and the second equation is called the Euler's equation. The Euler equation follows from the additive property of internal energy which is a homogeneous function of degree one. After light-cone reduction when we map non-relativistic variables in terms of relativistic ones, we see that at the leading order (ideal fluid), non-relativistic variables satisfy the following two equations,

$$
\begin{aligned}
\mathrm{d} \epsilon & =\tau \mathrm{d} s+\mu_{I} \mathrm{~d} q_{I}+\left(\epsilon+p-s \tau-q_{I} \mu_{I}\right) \frac{\mathrm{d} \rho}{\rho}, \\
2(\epsilon+p) & =s \tau+\mu_{I} q_{I} .
\end{aligned}
$$

Here we have identified the non-relativistic entropy density $s=S u^{+}$. The first equation, which follows from eq. (2.4), is the first law of thermodynamics satisfied by the nonrelativistic variables. The second, coming from eq. (2.6), is not exactly the Euler's equation for non-relativistic variables. We comment on this equation at the end of this sub-section.

As we consider the first derivative corrections to non-relativistic variables (evaluated in the previous sub-section), the first law of thermodynamics changes. However, that can not be the case. If we demand that our non-relativistic system is a physical one, then the first law should be satisfied by the non-relativistic variables at every order in derivative expansion. This demand forces us to add some higher derivative corrections to entropy density which we could not determine in section 4.23. Let's assume the following corrected forms:

$$
s=S u^{+}(1+\chi)
$$

$\chi$ is corresponding corrections.

Demanding first law of thermodynamics to be satisfied at first order in derivative we get:

$$
\begin{aligned}
& T S \mathrm{~d} \chi+M_{I} \mathrm{~d} \varpi_{I}-M_{I} \varpi_{I} \frac{\mathrm{d} E}{E+P}-\chi M_{I}\left(\mathrm{~d} Q_{I}-\frac{Q_{I}}{E+P} \mathrm{~d} E\right) \\
= & -\eta \frac{1}{d} \mathbf{Z} \frac{\mathrm{d} \mathbf{T}}{E+P}-\zeta \frac{\mathrm{d} E}{E+P} \frac{u^{\alpha} \nabla_{\alpha} E}{E+P}+\zeta \frac{\mathrm{d}(E-P)}{E+P} \frac{u^{\alpha} \nabla_{\alpha}(E-P)}{E+P} \\
& +\left[\chi S T+M_{I} \varpi_{I}+\eta \frac{d+2}{d} \mathbf{Z}-\zeta\left(\theta+2 \frac{u^{\alpha} \nabla_{\alpha} P}{E+P}\right)\right]\left(\frac{\mathrm{d} P}{E+P}+\frac{\mathrm{d} u^{+}}{u^{+}}\right) .
\end{aligned}
$$


Note that, thermodynamic relations (2.6)-(2.7), conservation equations (2.24)-(2.26) up to first order and all the identifications between non-relativistic and relativistic variables (obtained by LCR) have been extensively used to carry out the above computation. Now, using (2.24)-(2.26), we can write the above equation in the following form ${ }^{10}$

$$
T S u^{\mu} \nabla_{\mu} \chi+M_{I} u^{\mu} \nabla_{\mu} \varpi_{I}+M_{I} \varpi_{I} \theta=-\eta \frac{d+1}{2 d} \mathbf{Z}^{2}-\zeta \theta^{2}+\zeta\left(u^{+}\right)^{2}\left(\nabla_{k} v^{k}\right)^{2} .
$$

This equation fixes the correction to the entropy density.

The last equation in (4.65), which follows from Euler's equation (for the relativistic variables) does not imply that the non-relativistic energy density $\epsilon$ is a homogeneous function of degree one. In fact, this equation gets modified with the corrected values of non-relativistic entropy density. ${ }^{11}$ Therefore, we conclude that the non-relativistic system we obtain after light-cone reduction of a homogeneous relativistic fluid system, is not homogeneous any more (even at the leading order) i.e. the internal energy is not a homogeneous function of degree one.

\section{Non-relativistic entropy current}

The goal of this section is to write down an entropy current for a non-relativistic fluid system, and find constraints on various transport coefficients from the positivity of local entropy production. Using eq. (4.68) and properties of $\mathbf{Y}^{\mu \nu}$, one can reduce the eq. (2.36) and after a lengthy algebra, we get the following equation,

$$
\begin{aligned}
& \nabla_{+} s+\nabla_{i j} j_{S}^{i}+\nabla_{i}\left(\frac{\eta}{\tau\left(u^{+}\right)^{2}} \mathbf{Y}^{i}\right) \\
= & \frac{1}{\tau} \frac{n}{2} \sigma^{i j} \sigma_{i j}+\frac{1}{\tau} z\left(\nabla_{k} v^{k}\right)^{2}+\kappa\left[\frac{1}{\tau} \nabla^{i} \tau+\frac{q_{I} \tau}{2(\epsilon+p)} \nabla^{i}\left(\frac{\mu_{I}}{\tau}\right)-\frac{q_{I}\left(\epsilon_{I}^{i}-v_{k} \beta_{I}^{k i}\right)}{2(\epsilon+p)}\right]^{2} \\
& -\left[\frac{\epsilon_{I}^{i}-v_{k} \beta_{I}^{k i}}{\tau}-\nabla^{i}\left(\frac{\mu_{I}}{\tau}\right)\right] \frac{\xi_{J} q_{I}}{2(\epsilon+p)}\left[\frac{\epsilon_{J i}-v^{k} \beta_{J k i}}{\tau}-\nabla_{i}\left(\frac{\mu_{J}}{\tau}\right)\right] \\
& +\left[\frac{\epsilon_{I}^{i}-v_{k} \beta_{I}^{k i}}{\tau}-\nabla^{i}\left(\frac{\mu_{I}}{\tau}\right)\right]\left[\tilde{\sigma}_{I J} \tau \frac{\epsilon_{J i}-v^{k} \beta_{J k i}}{\tau}-\tilde{\xi}_{I J} \nabla_{i}\left(\frac{\mu_{J}}{\tau}\right)\right] \\
& -\left[\frac{\epsilon_{I}^{i}-v_{k} \beta_{I}^{k i}}{\tau}-\nabla^{i}\left(\frac{\mu_{I}}{\tau}\right)\right] \tilde{m}_{I} \nabla_{i} p+\left[\frac{\epsilon_{I}^{i}-v_{k} \beta_{I}^{k i}}{\tau}-\nabla^{i}\left(\frac{\mu_{I}}{\tau}\right)\right]\left\{\omega_{I} \Xi_{i}-\tilde{\omega}_{I J} \frac{2}{u^{+}} E_{J}^{j} \epsilon_{i j}\right\},
\end{aligned}
$$

where $j_{S}^{i}$ is the non-relativistic canonical entropy current defined as (similar to relativistic canonical entropy current given by eq. (2.34)),

$$
j_{S}^{i}=s v^{i}-\frac{\mu_{I}}{\tau} \varsigma_{I}^{i}
$$

\footnotetext{
${ }^{10}$ There will be two terms proportional to $\left\{C_{I J K} E_{J}^{\mu} B_{K \mu}\right\}$ which we have checked will vanish after reduction.

${ }^{11}$ Although the form of this equation can be maintained by adding some one derivative correction to temperature or chemical potential.
} 


\begin{tabular}{|c|c|c|}
\hline \multicolumn{2}{|c|}{ Non-rel. variables } & Rel. variables \\
\hline Velocity & $v^{i}$ & $\frac{u^{i}}{u^{+}}-\frac{\eta}{\left(u^{+}\right)^{2}(E+P)} \mathbf{Y}^{i}$ \\
\hline Mass Density & $\rho$ & $\left(u^{+}\right)^{2}(E+P)+\left(u^{+}\right)^{2}(\eta \mathbf{Z}-\zeta \theta)$ \\
\hline Energy Density & $\epsilon$ & $\frac{1}{2}(E-P)-\frac{1}{2}(\eta \mathbf{Z}-\zeta \theta)$ \\
\hline Pressure & $p$ & $P-\left(\frac{\eta}{d} \mathbf{Z}-\zeta \frac{u^{\alpha} \nabla_{\alpha} P}{E+P}\right)$ \\
\hline Charge & $q_{I}$ & $u^{+} Q_{I}-u^{+} \mho_{I} \epsilon^{i j} u^{+} \nabla_{i} v_{j}$ \\
\hline Entropy & $s$ & $S u^{+}(1+\chi)$ \\
\hline Scalar Potential & $\phi_{I}$ & $A_{I}^{-}$ \\
\hline Vector Potential & $a_{I}^{i}$ & $A_{I}^{i}$ \\
\hline
\end{tabular}

${ }^{a} \mathbf{Z}$ is given by eq. (2.28), $\mathbf{Y}^{i}$ is given by eq. (4.28) and $\chi$ is given by eq. (4.68).

Table 1. Relations between relativistic parameters and non-relativistic parameters (parity-even fluid)

We can rewrite eq. (5.1) in more conventional form as

$$
\begin{aligned}
\nabla_{+} s+\nabla_{i} j_{S}^{i}= & \frac{1}{\tau} \frac{n}{2} \sigma^{i j} \sigma_{i j}+\frac{1}{\tau} z\left(\nabla_{k} v^{k}\right)^{2}+\frac{1}{\tau} \nabla^{i}\left[\kappa \nabla_{i} \tau+\tau \sigma_{I} \nabla^{i}\left(\frac{\mu_{I}}{\tau}\right)-\sigma_{I}\left(\epsilon_{I}^{i}-v_{k} \beta_{I}^{k i}\right)\right] \\
& +\left[\frac{\epsilon_{J i}-v^{k} \beta_{J k i}}{\tau}-\nabla_{i}\left(\frac{\mu_{J}}{\tau}\right)\right] \varsigma_{I}^{i} .
\end{aligned}
$$

The first three terms on the right hand side survive for uncharged dissipative fluid [1]. Here we compute the other terms which are responsible for local entropy production for parity-odd charged fluid in presence of background fields. Since we demand that the fluid satisfies the second law of thermodynamics, the right hand side of eqn. (5.1) should be positive definite. This implies following constraints on the transport coefficients

$$
\begin{gathered}
\tilde{m}_{I}=0, \quad n \geq 0, \quad z \geq 0, \quad \tilde{\sigma}_{I J}=\frac{1}{\tau} \tilde{\xi}_{I J}, \\
{\left[\tilde{\xi}_{I J}-\frac{\xi_{J} q_{I}}{2(\epsilon+p)}\right] \quad \text { matrix is positive definite. }}
\end{gathered}
$$

These constraints are compatible with what we get from relativistic entropy production condition. However the last term in eq. (5.1) is a parity odd term and can not be written as a perfect square. Therefore, it seems that the presence of this term breaks the second law of thermodynamics. However, similar to the relativistic fluid dynamics, we can add extra parity-odd terms (allowed by symmetry) to entropy current to make right hand side a perfect square.

Note that the last term in eq. (5.1) appears only in $2+1$ dimensions. Since the first order parity-odd terms in eq. (5.1) do not appear in the non-relativistic fluid theories in 


\begin{tabular}{|c|c|c|}
\hline \multicolumn{2}{|c|}{ Non-rel. variables } & Rel. variables \\
\hline $\begin{array}{l}\text { Bulk Viscos- } \\
\text { ity }\end{array}$ & $z$ & $\zeta u^{+}$ \\
\hline $\begin{array}{l}\text { Shear Vis- } \\
\text { cosity }\end{array}$ & $n$ & $\eta u^{+}$ \\
\hline $\begin{array}{l}\text { Electrical } \\
\text { Conductiv- } \\
\text { ity }\end{array}$ & $\tilde{\sigma}_{I J}$ & $\lambda_{I J} u^{+}+\eta u^{+} Q_{I} Q_{J} /(E+P)^{2}$ \\
\hline \multirow[t]{2}{*}{$\begin{array}{l}\text { Thermal } \\
\text { Conductiv- } \\
\text { ity }\end{array}$} & $\kappa$ & $2 n(\epsilon+p) / \tau \rho$ \\
\hline & $\tilde{\kappa}_{I}$ & $\kappa q_{I} / 2(\epsilon+p)$ \\
\hline $\begin{array}{l}\text { Charge Con- } \\
\text { ductivity }\end{array}$ & $\sigma_{I}$ & $n q_{I} / \rho$ \\
\hline $\begin{array}{l}\text { Momentum } \\
\text { Current }\end{array}$ & $t^{i j}$ & $v^{i} v^{j} \rho+p g^{i j}-n \sigma^{i j}-z g^{i j} \nabla_{k} v^{k}$ \\
\hline $\begin{array}{l}\text { Energy Cur- } \\
\text { rent }\end{array}$ & $j^{i}$ & $\begin{array}{c}v^{i}\left(\epsilon+p+\frac{1}{2} \rho \mathbf{v}^{2}\right) \\
-n \sigma^{i k} v_{k}-z v^{i} \nabla_{k} v^{k}-\kappa \nabla^{i} \tau \\
-\tau \sigma_{I} \nabla^{i}\left(\frac{\mu_{I}}{\tau}\right)+\sigma_{I}\left(\epsilon_{I}^{i}-v_{j} \beta_{I}^{j i}\right)\end{array}$ \\
\hline $\begin{array}{l}\text { Charge Cur- } \\
\text { rent }\end{array}$ & $j_{I}^{i}$ & $\begin{array}{c}q_{I} v^{i}-\tilde{\kappa}_{I} \nabla^{i} \tau-\tau \tilde{\sigma}_{I J} \nabla^{i}\left(\frac{\mu_{J}}{\tau}\right) \\
+\tilde{\sigma}_{I J}\left(\epsilon_{J}^{i}-v_{k} \beta_{J}^{k i}\right)\end{array}$ \\
\hline $\begin{array}{l}\text { Entropy } \\
\text { Current }\end{array}$ & $j_{S}^{i}$ & $\begin{array}{l}s v^{i}+\frac{\mu_{I}}{\tau}\left(\tilde{\kappa}_{I} \nabla^{i} t+\tau \tilde{\sigma}_{I J} \nabla^{i}\left(\frac{\mu_{J}}{\tau}\right)\right. \\
\left.-\tilde{\sigma}_{I J}\left(\epsilon_{J}^{i}-v_{k} \beta_{J}^{k i}\right)\right)\end{array}$ \\
\hline
\end{tabular}

Table 2. Relations between relativistic and non-relativistic transport coefficients, energy momentum tensors and currents (parity-even fluid)

more than two spatial dimensions, we have a complete and consistent description ${ }^{12}$ for these theories. We summarize the results for them in tables 1,2 .

\subsection{Parity-odd corrections to NR entropy current}

For fluids in two spatial dimensions however, we must add some parity-odd terms to the entropy current with some arbitrary transport coefficients and determine them from the condition that there is no local entropy loss during the flow of the system. We make the most generic parity-odd modification to the entropy current in two spatial dimensions as follows

$$
j_{S}^{i} \rightarrow j_{S}^{i}+\left\{\mathfrak{a} \epsilon^{i j} \nabla_{j} \tau+\mathfrak{b}_{I} \epsilon^{i j} \nabla_{j}\left(\frac{\mu_{I}}{\tau}\right)+\mathfrak{c} \epsilon^{i j} \nabla_{j} p+\mathfrak{d}_{I} \epsilon^{i j} \epsilon_{I j}+\mathfrak{f}_{I} \epsilon^{i j} v^{k} \beta_{I k j}\right\} .
$$

\footnotetext{
${ }^{12}$ This is the description of a non-relativistic fluid obtained from the most generic relativistic fluid.
} 
$\mathfrak{a}, \mathfrak{b}_{I}, \mathfrak{c}, \mathfrak{d}_{I}$ and $\mathfrak{f}_{I}$ are arbitrary transport coefficients. After adding these extra terms the right hand side of eq. (5.1) can be written as (parity-odd part only)

$$
\begin{aligned}
& \left\{\nabla_{i}\left(\mathfrak{a} \epsilon^{i j} \nabla_{j} \tau+\mathfrak{b}_{I} \epsilon^{i j} \nabla_{j}\left(\frac{\mu_{I}}{\tau}\right)+\mathfrak{c} \epsilon^{i j} \nabla_{j} p+\mathfrak{d}_{I} \epsilon^{i j} \epsilon_{I j}+\mathfrak{f}_{I} \epsilon^{i j} v^{k} \beta_{I k j}\right)\right\} \\
& +\left[\frac{1}{T} E_{I}^{i}-\nabla^{i}\left(\frac{\mu_{I}}{\tau}\right)\right]\left\{\omega_{I} \Xi_{i}-\tilde{\omega}_{I J} \frac{2}{u^{+}} E_{J}^{j} \epsilon_{i j}\right\} \\
= & \left\{\epsilon^{i j} \nabla_{j} \tau\right\} \nabla_{i} \mathfrak{a}+\left\{\epsilon^{i j} \nabla_{j}\left(\frac{\mu_{I}}{\tau}\right)\right\} \nabla_{i} \mathfrak{b}_{I}+\left\{\epsilon^{i j} \nabla_{j} p\right\} \nabla_{i} \mathfrak{c} \\
& +\left\{\epsilon^{i j} \epsilon_{I j}\right\} \nabla_{i} \mathfrak{d}_{I}+\left\{\frac{1}{2} v^{k} \epsilon^{i j} \beta_{I i j}\right\} \nabla_{k} \mathfrak{f}_{I} \\
& -\left\{\epsilon^{i j} \nabla_{i}\left(\frac{\mu_{I}}{\tau}\right)\right\}\left(\frac{\omega_{I}}{\tau\left(u^{+}\right)^{2}} \nabla_{j} \tau+\omega_{I} \frac{q_{I} \tau}{\rho} \nabla_{j}\left(\frac{\mu_{I}}{\tau}\right)-\frac{2 \omega_{I}}{\rho} \nabla_{j} p\right) \\
& +\left\{\epsilon^{i j} \frac{1}{T} E_{I i}\right\}\left(\frac{\omega_{I}}{\tau\left(u^{+}\right)^{2}} \nabla_{j} \tau+\frac{\tau}{\rho}\left(\omega_{I} q_{J}+\omega_{J} q_{I}-2 \rho \tilde{\omega}_{J I}\right) \nabla_{j}\left(\frac{\mu_{J}}{\tau}\right)-\frac{2 \omega_{I}}{\rho} \nabla_{j} p\right) \\
& +\left\{\epsilon^{i j} \frac{1}{T} E_{I i}\right\}\left(\frac{\omega_{I}}{\rho} Q_{J} E_{J j}-\tilde{\omega}_{I J} \frac{2}{u^{+}} E_{J j}\right)+\frac{1}{2}\left\{\mathfrak{f}_{I} \epsilon^{i j} \beta_{I i j} \nabla_{k} v^{k}-\mathfrak{d}_{I} \epsilon^{i j} \nabla_{+} \beta_{I i j}\right\} \\
= & 0 .
\end{aligned}
$$

To obey the second law of thermodynamics we claim that the coefficients of all the independent terms must vanish. This is possible except for the last two terms, for which we get the following sets of constraints:

$$
\begin{aligned}
\frac{\partial \mathfrak{a}}{\partial p}-\frac{\partial \mathfrak{c}}{\partial \tau} & =0 \\
\frac{\partial \mathfrak{a}}{\partial\left(\mu_{I} / \tau\right)}-\frac{\partial \mathfrak{b}_{I}}{\partial \tau} & =\frac{\epsilon+p}{\tau} \frac{2 \omega_{I}}{\rho} \\
\frac{\partial \mathfrak{b}_{I}}{\partial p}-\frac{\partial \mathfrak{c}}{\partial\left(\mu_{I} / \tau\right)} & =\frac{2 \omega_{I}}{\rho} \\
-\frac{\partial \mathfrak{f}_{I}}{\partial \tau} & =\frac{\partial \mathfrak{d}_{I}}{\partial \tau}=\frac{\epsilon+p}{\tau^{2}} \frac{2 \omega_{I}}{\rho} \\
\frac{\partial \mathfrak{f}_{I}}{\partial p} & =-\frac{\partial \mathfrak{d}_{I}}{\partial p}=\frac{2 \omega_{I}}{\tau \rho} \\
-\frac{\partial \mathfrak{f}_{I}}{\partial\left(\mu_{J} / \tau\right)} & =\frac{\partial \mathfrak{d}_{I}}{\partial\left(\mu_{J} / \tau\right)}=\frac{1}{\rho}\left(\omega_{I} q_{J}+\omega_{J} q_{I}-2 \rho \tilde{\omega}_{J I}\right)
\end{aligned}
$$

additionally, the following matrices must be symmetric:

$$
\begin{array}{r}
2 \rho \tilde{\omega}_{I J}-\omega_{I} q_{J} \\
\frac{\rho}{t} \frac{\partial \mathfrak{b}_{J}}{\partial\left(\mu_{I} / t\right)}-\omega_{I} q_{J}
\end{array}
$$

It should be noted that the new coefficients introduced in $j_{S}^{i}$ need not be most generic, and their only significance is to make the parity-odd terms in entropy current vanish. Hence, a minimal choice is as follows:

$$
\mathfrak{a}=\mathfrak{c}=0, \quad \mathfrak{f}_{I}=-\mathfrak{d}_{I},
$$


which solves the respective constraints, and leaves us only two independent coefficients in entropy current: $\mathfrak{b}_{I}$ and $\mathfrak{d}_{I}$.

Now we turn attention towards the last two terms of eq. (5.6). They vanish if we consider the fluid is incompressible and placed in a time independent background. For compressible fluid the second last term is non-zero and can be negative. Hence the second law implies that the $\mathfrak{f}_{I}=\mathfrak{d}_{I}=0$. As a result all the parity-odd terms vanish for compressible fluid. The resultant fluid is completely parity-even and we summarize the results in tables 1, 2. On the other hand if we consider the background field is time dependent, then the last term can violate the second law. However, in that case, one has to be careful about the change of entropy of the background also. The second law should hold for the fluid and the background system together. The last term produces some vortex kind of motion in the fluid. In that case the velocity of fluid particles around those vortexes becomes very large and our analysis (derivative expansion) may break down.

It should be noted that we demand our non-relativistic system to obey the second law of thermodynamics. We start with a relativistic fluid but never impose any physicality constraint (except the fluid satisfies first law of thermodynamics) on the relativistic side. Relativistic system, in our case, can be considered as a mathematical model (where we allow all possible terms allowed by the symmetry). After reduction we see that at least for the parity-even sector constraint from the second law turns out to be same (compatible) both in relativistic and non-relativistic case (eq. (2.37) and (5.4)). But for parity-odd sectors they are completely different. For example, $C_{I J K}$ do not even appear in non-relativistic relations eq. (5.7)-(5.9), though for relativistic fluid the second law relates $\mho_{I}$ and $\tilde{\mho}_{I J}$ to $C_{I J K}$ (eq. (2.40)). Whereas for non-relativistic fluid one can consider $\omega_{I}$ and $\tilde{\omega}_{I J}$ to be independent and $\mathfrak{f}_{I}$ and $\mathfrak{d}_{i}$ are fixed in terms of them. However, one can also start with a relativistic fluid satisfying second law of thermodynamics (as an additional condition). In that case for an incompressible fluid it is possible to express the non-relativistic transport coefficients $\left(\mathfrak{f}_{I}, \mathfrak{d}_{I}, \omega_{I}, \tilde{\omega}_{I}\right)$ in terms of anomaly coefficients of the relativistic system. But for compressible fluid it is not possible as these coefficients turn out to be zero.

\subsection{Incompressible fluid in constant magnetic background in (2+1)-dim}

Equation of state of an incompressible fluid is given by:

$$
\rho=\text { constant, }
$$

which implies that $\nabla_{k} v^{k}=0$, or in other words, there is no compression or expansion of the fluid during the flow.

Using the non-relativistic constitutive equations one can easily show that:

$$
\frac{\mathrm{d} \epsilon}{\mathrm{d} t}+(\epsilon+p) \nabla_{k} v^{k}=v_{j} \nabla_{i} \pi^{i j}-\nabla_{i} \varsigma^{i},
$$

which means that for incompressible fluids, $\mathrm{d} \epsilon / \mathrm{d} t$ is atmost two derivative in order, and is zero for ideal fluids. Considering this result at first derivative order we can infer that:

$$
u^{\mu} \nabla_{\mu}(E-P)=0 \Rightarrow \frac{u^{\mu} \nabla_{\mu} P}{E+P}=-\theta,
$$

in first derivative order. Same can be inferred directly from eq. (4.32). 
Maxwell's equation in the magnetic limit appendix A are given by:

$$
\begin{aligned}
\nabla_{i} \epsilon_{I}^{i} & =0, \\
\nabla_{i} \beta_{I}^{i j} & =0, \\
2 \epsilon^{i j} \nabla_{i} \epsilon_{I j}+\epsilon^{i j} \nabla_{+} \beta_{I i j} & =0 .
\end{aligned}
$$

Second equation implies that magnetic field is constant in space. Also, if magnetic field $\beta_{I}^{12}$ is time independent (as we demand), electric field is curl free. But first equation already tells us that electric field should be divergence-free. Hence electric field is constant over the 2 dimensional space. However, electric field can still be time dependent, as the corresponding term does not appear in the magnetic limit of Maxwell's equations. Finally in our case we have, a constant electric field (may depend on time) and a constant magnetic field.

For this system, the form of $j_{S}^{i}$ is given by

$$
j_{S}^{i}=s v^{i}-\frac{\mu_{I}}{\tau} v_{I}^{i}+\mathfrak{b}_{I} \epsilon^{i j} \nabla_{j}\left(\frac{\mu_{I}}{\tau}\right)+\mathfrak{d}_{I} \epsilon^{i j}\left(\epsilon_{I j}-v^{k} \beta_{I k j}\right) .
$$

where $\mathfrak{b}_{I}$ and $\mathfrak{d}_{I}$ are determined by equations (5.7)-(5.9). Hence we will get the positivity of entropy in the form of eq. (5.3), along with constraints eq. (5.4). However $\omega_{I}$ and $\tilde{\omega}_{I J}$ remain unconstrained by entropy positivity. Results of this case have been summarized in tables 3 .

\section{Discussion}

We started with a generic $(d+2)$-dim non-conformal relativistic fluid $(d \geq 2)$ with anomalies specific to $d=2$ in presence of background electromagnetic fields on flat space. Light cone reduction of this system gives a $(d+1)$-dim non-relativistic fluid. We demand that our non-relativistic fluid satisfies the second law of thermodynamics which imposes certain constraints on the system. For example we find that the parity odd terms in non-relativistic theory can only sustain for incompressible fluid in electromagnetic background with constant magnetic field in $(2+1)$-dim.

Our non-relativistic fluid (obtained by LCR) has generic ${ }^{13}$ dissipative terms, which are allowed by the symmetry and the condition that local entropy production is always positive definite. LCR does not constraint the size of these coefficients. They all are fixed in terms of the transport coefficients of the 'mother' relativistic theory. On the contrary, when one performs a $1 / c$ expansion the relativistic constitutive equations to get a nonrelativistic fluid system, as done in [15], many of these terms are suppressed depending on the physical considerations and the type of system under view. We discuss the basic aspects of $1 / c$ expansion in appendix $\mathrm{B}$.

Apart from the dissipative terms, our system and the system obtained by $1 / c$ expansion in [15] have certain fundamental differences. Firstly, their system is 'extensive' as the

\footnotetext{
${ }^{13}$ Word generic here just means all possible terms obtained by LCR, and not all possible terms allowed by symmetry. We might have more or less terms, depending on the type of relativistic theory we are starting with and the identification.
} 


\begin{tabular}{|c|c|c|}
\hline \multicolumn{2}{|c|}{ Non-rel. variables } & Rel. variables \\
\hline $\begin{array}{c}\text { Parity-odd } \\
\text { coefficients }\end{array}$ & $\begin{array}{l}\omega_{I} \\
\tilde{\omega}_{I J}\end{array}$ & $\begin{array}{c}\mho_{I}\left(u^{+}\right)^{2} \\
\tilde{\mho}_{I J} u^{+}\end{array}$ \\
\hline Mom. Curr. & $t^{i j}$ & $v^{i} v^{j} \rho+p g^{i j}-n \sigma^{i j}$ \\
\hline $\begin{array}{l}\text { Energy } \\
\text { Current }\end{array}$ & $j^{i}$ & $\begin{array}{c}v^{i}\left(\epsilon+p+\frac{1}{2} \rho \mathbf{v}^{2}\right)-n \sigma^{i k} v_{k} \\
-\kappa \nabla^{i} \tau-\tau \sigma_{I} \nabla^{i}\left(\frac{\mu_{I}}{\tau}\right)+\sigma_{I}\left(\epsilon_{I}^{i}-v_{j} \beta_{I}^{j i}\right)\end{array}$ \\
\hline $\begin{array}{l}\text { Charge } \\
\text { Current }\end{array}$ & $j_{I}^{i}$ & $\begin{array}{c}q v^{i}-\tilde{\kappa}_{I} \nabla^{i} \tau-\tau \tilde{\sigma}_{I J} \nabla^{i}\left(\frac{\mu_{J}}{\tau}\right) \\
+\tilde{\sigma}_{I J}\left(\epsilon_{J}^{i}-v_{k} \beta_{J}^{k i}\right) \\
+\omega_{I} \epsilon^{i j}\left(\frac{\kappa}{n} \nabla_{j} \tau+\frac{\sigma_{J} \tau}{n} \nabla_{j}\left(\frac{\mu_{J}}{\tau}\right)-\frac{2}{\rho} \nabla_{j} p\right) \\
+\bar{\sigma}_{I J} \epsilon^{i j}\left(\epsilon_{J j}-v^{k} \beta_{J k j}\right)\end{array}$ \\
\hline $\begin{array}{l}\text { Entropy } \\
\text { Current }\end{array}$ & $j_{S}^{i}$ & $\begin{array}{c}s v^{i}+\frac{\mu_{I}}{\tau}\left(\tilde{\kappa}_{I} \nabla^{i} \tau+\tau \tilde{\sigma}_{I J} \nabla^{i}\left(\frac{\mu_{J}}{\tau}\right)\right. \\
\left.-\tilde{\sigma}_{I J}\left(\epsilon_{J}^{i}-v_{k} \beta_{J}^{k i}\right)\right) \\
-\frac{\mu_{I}}{\tau} \omega_{I} \epsilon^{i j}\left(\frac{\kappa}{n} \nabla_{j} \tau-\frac{2}{\rho} \nabla_{j} p\right) \\
+\left(\mathfrak{b}_{J}-\frac{\omega_{I} \mu_{I} q_{J}}{\rho}\right) \epsilon^{i j} \nabla_{j}\left(\frac{\mu_{J}}{\tau}\right) \\
+\left(\mathfrak{d}_{J}-\frac{\mu_{I}}{\tau} \bar{\sigma}_{I J}\right) \epsilon^{i j}\left(\epsilon_{J j}-v^{k} \beta_{J k j}\right)\end{array}$ \\
\hline
\end{tabular}

Table 3. Relativistic and non-relativistic transport coefficients for parity-odd fluid

thermodynamic variables follow Euler's relation; however since LCR breaks the Euler's relation, our system is no longer extensive. Secondly, in our system $\rho$ is not necessarily proportional to $q_{I}$, while in [15] it is true at least at the leading $1 / c$ order. This is why our non-relativistic system has one more independent parameter as opposed to the $1 / c$ case. We can however enforce $\rho \propto q_{I}$ in our system as well, but it turns out that demanding so switches off all the dissipative terms from the theory except for the bulk viscosity.

In [15] authors do not present an entropy current calculation for non-relativistic fluid obtained by $1 / c$ expansion. In fact, as we will review in appendix B, the entropy positivity turns out to be trivial, and is just followed from the leading order entropy current of the relativistic theory. The constraints on the transport coefficients (which survives at the leading order) also turns out to be the same. However in our case, we have slightly different constraints, because of the above mentioned fundamental differences between the two cases.

\section{Acknowledgments}

We would like to acknowledge Pratik Roy and Rahul Soni for valuable discussions. SD would like to thank the hospitality of IACS, Kolkata where part of this work has been done. NB would like to thank the DST Ramanujan Fellowship. Finally we are indebted 
to the people of India for their unconditional support towards research and development in basic science.

\section{A Magnetic limit of electrodynamics}

Maxwell's Electrodynamics is a relativistic theory. In fact it was a precursor to Einstein's Special Theory of Relativity. Having a consistent relativistic description of electrodynamics, eradicated any need for a 'non-relativistic' theory of electrodynamics. However as in current context, one needs a Galilean description of electrodynamics, just to keep it consistent with other non-relativistic theories.

Recently in [18], authors discussed the non-relativistic limit of electrodynamics in two distinct ways. Depending on the strength of fields, ${ }^{14}$ we can have two discrete nonrelativistic limits: $|\vec{E}| \gg c|\vec{B}|$ electric limit and $|\vec{E}| \ll c|\vec{B}|$ magnetic limit. We find that the light-cone reduction of relativistic sourceless Maxwell's equations, under certain identifications, gives magnetic limit of Maxwell's equations, which will be our interest of discussion here. A more thorough discussion of non-relativistic electrodynamics can be found in [18].

In arbitrary dimensions, inhomogeneous Maxwell's equations are given by:

$$
\nabla_{i} F^{i 0}=-\mu_{o} c \rho, \quad \nabla_{0} F^{0 i}+\nabla_{j} F^{j i}=-\mu_{o} J^{i},
$$

while the homogeneous ones (Bianchi identities) are:

$$
\epsilon^{\alpha \beta \cdots \mu \nu \sigma} \nabla_{\mu} F_{\nu \sigma}=0,
$$

where $\epsilon^{\alpha \beta \cdots \mu \nu \sigma}$ is the full-rank Levi-Cevita tensor. The last identity follows directly from the gauge invariant form of $F^{\mu \nu}=\nabla^{\mu} A^{\nu}-\nabla^{\nu} A^{\mu}$ or the constituent fields: ${ }^{15} E^{i}=-\nabla^{i} \phi-\nabla_{0} A^{i}$ and $F^{i j}=\nabla^{i} A^{j}-\nabla^{j} A^{i}$. As LCR gives a non-relativistic theory with gauge invariance, we are interested in a non-relativistic limit which preserves eq. (A.2). We will see that the magnetic limit essentially does the same. However there exists a consistent electric limit as well, where Bianchi identities are broken, but inhomogeneous Maxwell's equations are preserved.

In terms of dimensionless fields and sources one can write the inhomogeneous Maxwell's equations as:

$$
\alpha \nabla_{i} E^{i}=\rho, \quad \beta \frac{\partial}{\partial t} E^{i}+\nabla_{j} F^{j i}=-\frac{\beta}{\alpha} J^{i},
$$

and the Bianchi identities as:

$$
\begin{aligned}
\epsilon_{l m \cdots i j} \nabla^{i} E^{j} & =-\beta \frac{1}{2} \epsilon_{l m \cdots i j} \frac{\partial}{\partial t} F^{i j}, \\
\epsilon^{m \cdots k i j} \nabla_{k} F_{i j} & =0,
\end{aligned}
$$

\footnotetext{
${ }^{14}$ In this section we use the conventional notation for electrodynamics: $E$ for electric field, $B$ for magnetic field, $\rho$ for charge density, $J$ for charge current etc.

${ }^{15}$ Instead of the conventional magnetic field, we use its 2 nd rank dual in our work, as in arbitrary dimensions we do not have electromagnetic duality and magnetic field does not have any fixed rank, while its dual has.
} 
where $\alpha$ and $\beta$ are some dimensionless constants given by:

$$
\alpha=\frac{[E] \epsilon_{o}}{[\rho][L]}, \quad \beta=\frac{[L]}{[T] c} .
$$

From here we can easily read out, that in non-relativistic limit $\beta \ll 0$. To preserve the Bianchi identities therefore, $E^{i}$ should be one order smaller than $F^{i j}$; which is why this limit is called 'magnetic limit'. If we measure smallness in terms of a parameter $\epsilon \sim 1 / c$, we will have $E^{i} \sim \epsilon^{n+1}$ and $F^{i j} \sim \epsilon^{n}$. Therefore from eq. (A.3), first nontrivial order of $\rho \sim \alpha \epsilon^{n+1}$, and $J^{i} \sim \alpha \epsilon^{n-1}$, and inhomogeneous Maxwell's Equations (in conventional units) reduce to:

$$
\nabla_{i} E^{i}=\frac{\rho}{\epsilon_{o}}, \quad \nabla_{j} F^{j i}=-\mu_{o} J^{i} .
$$

Essentially we have just dropped the displacement current term from the Ampere's Law. To explicitly see if this limit is Galilean invariant, one can lookup [18]. These are the very equations that have been used in section 4.1 and 5.2.

One can now push the expansion of eq. (A.3) to $\epsilon^{n+2}$ order and derive the continuity equation:

$$
\frac{\partial}{\partial t} \rho+\nabla_{i} J^{i}=0
$$

It should be remembered however that there are mixed orders in this equation and to the highest order it just states: $\nabla_{i} J^{i}=0$, which also follows from eq. (A.7). Continuity equation takes its usual form only when first two leading orders of $J^{i}$ vanish and $\rho \sim J^{i}$, but in which case one of the Maxwell's equations modifies to: $\nabla_{j} F^{j i}=0$. This in conjugation with the Bianchi identity would mean that the magnetic field is constant over space.

Finally in conventional units this limit can be summarized as: $F^{i j} \sim E^{i} \sim c^{-n}$, and to the maximum order $\rho \sim c^{-n} \epsilon_{o}, J^{i} \sim c^{-n+2} \epsilon_{o}$.

\section{B 1/c expansion of relativistic fluid dynamics}

In this section we discuss briefly the $1 / c$ expansion limit of relativistic fluid dynamics to get a non-relativistic theory, using the prescription of [15]. We consider here just a parity-even fluid for comparison with our work. Parity-odd sector in our case and in [15] are in different hydrodynamic frames and thus are incomparable.

The constitutive equations of a relativistic fluid (with appropriate factors of $c$ ) are:

$$
\nabla_{\mu} T^{\mu \nu}=c F_{I}^{\nu \alpha} J_{I \alpha}, \quad \nabla_{\mu} J_{I}^{a \mu}=0,
$$

where

$$
\begin{aligned}
& T^{\mu \nu}=(E+P) u^{\mu} u^{\nu}+P g^{\mu \nu}+\Pi^{\mu \nu}, \\
& J_{I}^{a \mu}=Q_{I} u^{\mu}+\mathrm{U}_{\mathrm{I}}^{\mu} .
\end{aligned}
$$

Respective dissipative terms are given as:

$$
\begin{aligned}
\Pi^{\mu \nu} & =-2 \eta \tau^{\mu \nu}-\zeta \theta P^{\mu \nu} \\
\Upsilon_{I}^{\mu} & =-\frac{1}{c^{2}} T \lambda_{I J} P^{\mu \nu} \nabla_{\nu}\left(\frac{M_{J}}{T}\right)+\frac{1}{c} \lambda_{I J} E_{J}^{\mu} .
\end{aligned}
$$


We can separate out rest contributions from $E$ and $M_{I}$ :

$$
E=R c^{2}+\mathcal{E}, \quad M_{I}=\frac{1}{\mathcal{K}} m_{I} c^{2}+\mu_{I}
$$

Here $m_{I}$ is the mass is to ' $I$ 'th charge ratio of constituent particles in their local rest frame, which is assumed to be constant. $\mathcal{K}$ is the total number of $\mathrm{U}(1)$ charges. Non-relativistic mass and energy density are related to their relativistic counterparts as:

$$
\rho=R \Gamma, \quad \epsilon=\mathcal{E} \Gamma,
$$

where $\Gamma=\left(1-\mathbf{v}^{2} / c^{2}\right)^{-1}$. Since the fluid under consideration is single component, $R / Q_{I}=$ $m_{I}$ is a constant. Non-relativistic charge density is defined as:

$$
q_{I}=J_{I}^{0} .
$$

Pressure and Temperature are however kept same, which to be consistent with the main text notation we will denote as: $\tau=T$ and $p=P$.

Before continuing with expansion we need to fix the order of various quantities. $\rho, \epsilon, p, \tau, v^{i}$ can be thought of finite order without much ambiguity. $m_{I}$ on the other hand is quite sensitive to the kind of system under consideration. Let us consider a charged fluid made of 'ions' where $m_{I} \sim c^{2} ; m_{I}$ cannot be too low, or else the fluid would start coupling to the background fields. Correspondingly the charge density $q_{I}$ would be of order $c^{-2}$. Further, to keep the thermodynamics intact, one has to assume $\mu_{I}$ to be of order of $c^{2}$. Finally, for external fields to have finite effect (e.g. force) on the fluid, $\epsilon_{I}^{i} \sim \beta_{I}^{i j} \sim c^{2}$.

Using this information we can reduce the energy-momentum tensor to:

$$
\begin{aligned}
T^{00} & =\rho c^{2}+\frac{1}{2} \rho \mathbf{v}^{2}+\epsilon+\mathcal{O}(1 / c), \\
T^{i 0} & =\rho v^{i} c+\left(\frac{1}{2} \rho \mathbf{v}^{2}+\epsilon+p\right) \frac{v^{i}}{c}+\frac{1}{c} \pi^{i j} v_{j}+\mathcal{O}\left(1 / c^{2}\right), \\
T^{i j} & =t^{i j}+\mathcal{O}(1 / c), \quad t^{i j}=\rho v^{i} v^{j}+p g^{i j}+\pi^{i j},
\end{aligned}
$$

where we have used:

$$
\begin{aligned}
n & =\frac{\eta}{c}, \quad z=\frac{\zeta}{c} \\
\pi^{i j} & =-n\left(\nabla^{i} v^{j}+\nabla^{j} v^{i}-g^{i j} \frac{2}{d} \nabla_{k} v^{k}\right)-z g^{i j} \nabla_{k} v^{k} .
\end{aligned}
$$

Energy-momentum conservation equations, at highest order, will then reduce to:

$$
\begin{aligned}
\partial_{t} \rho+\partial_{i}\left(\rho v^{i}\right) & =0, \\
\partial_{t}\left(\rho v^{i}\right)+\partial_{i} t^{i j} & =\epsilon_{I}^{i} q_{I}+\beta_{I}^{i j} j_{I j} .
\end{aligned}
$$

It will be worth to mention here the underlying assumption: $n, z \sim 1$ which is just an empirical fact. This is precisely the reason why no dissipative corrections appear to the continuity equation. We can now expand the charge current:

$$
\begin{aligned}
J_{I}^{0} & =q_{I}, & q_{I} & =Q_{I} \Gamma+\mathcal{O}\left(1 / c^{6}\right), \\
J_{I}^{i} & =\frac{1}{c} j_{I}^{i}+\mathcal{O}\left(1 / c^{7}\right), & j_{I}^{i} & =q_{I} v^{i}+\varsigma_{I}^{i},
\end{aligned}
$$


where we have used:

$$
\begin{aligned}
\tilde{\sigma}_{I J} & =\frac{\lambda_{I J}}{c}, \quad \tilde{\gamma}_{I J}=\frac{\gamma_{I J}}{c}, \\
\varsigma_{I}^{i} & =c^{2} \tilde{\sigma}_{I J} \frac{m_{J}}{\mathcal{K}_{\tau}} \nabla^{i} \tau .
\end{aligned}
$$

Here again we have used a physical input to fix the order of $\tilde{\sigma}_{I J}$. We would demand that in the non-relativistic theory, charge to mass ratio should be a constant. Therefore charge continuity and mass continuity equations should be the same upto leading order in $1 / c$, and hence $\varsigma_{I}^{i}$ should at maximum be of the order of $c^{-4}$. It further implies that $\tilde{\sigma}_{I J} \sim c^{-8}$. As a consequence, effects like electric conductivity are suppressed in whole theory.

Finally we use: $\nabla_{\mu}\left(T^{\mu 0}-\frac{1}{\mathcal{K}} m_{I} c^{2} J_{I}^{\mu}\right)=0$ to get the energy conservation:

$$
\begin{aligned}
\partial_{t}\left[\frac{1}{2} \rho \mathbf{v}^{2}+\epsilon\right]+\partial_{i}\left[v^{i}\left(\frac{1}{2} \rho \mathbf{v}^{2}+\epsilon+P\right)+\varsigma^{i}\right] & =0, \\
\varsigma^{i} & =\pi^{i j} v_{j}-\kappa \nabla^{i} \tau,
\end{aligned}
$$

where we have identified the thermal conductivity:

$$
\kappa=\tilde{\sigma}_{I J} \frac{c^{4}}{\mathcal{K}^{2}} \frac{m_{I}^{a} m_{J}^{a}}{\tau} .
$$

which is of finite order.

The non-relativistic theory has essentially turned out to be identical to the chargeless fluid discussed in [1], because for uni-component fluids, charge density serves just as number density upto a factor. For multi-particle fluids however this might become more interesting, as then one does not have to expect charge is to mass ratio to be constant. In fact one can have some chargeless particles also in the system.

\section{B.1 1/c expansion of thermodynamics}

First law of thermodynamics and the Euler's relation in the relativistic theory are given by:

$$
\begin{aligned}
\mathrm{d} E & =T \mathrm{~d} S+M_{I} \mathrm{~d} Q_{I}, \\
E+P & =T S+M_{I} Q_{I} .
\end{aligned}
$$

Under $1 / c$ expansion they reduce to:

$$
\begin{aligned}
\mathrm{d} \epsilon & =\epsilon \mathrm{d} s+\mu_{I} \mathrm{~d} q_{I}, \\
\epsilon+p & =\tau s+\mu_{I} q_{I},
\end{aligned}
$$

which are just the non-relativistic analogues of the same equations. We therefore conclude that the non-relativistic system gained by $1 / c$ expansion follows extensivity.

Now lets have a look at the second law of thermodynamics in the relativistic side, which mentions: $\nabla_{\mu} J_{S}^{\mu} \geq 0$. From eq. (2.35) we know the positive definite form of entropy current:

$$
\nabla_{\mu} J_{S}^{\mu}=\frac{1}{T} \eta \tau^{\mu \nu} \tau_{\mu \nu}+\frac{1}{T} \zeta \theta^{2}+\left[\frac{E_{I}^{\alpha}}{c^{2} T}-\frac{1}{c} P^{\alpha \mu} \nabla_{\mu}\left(\frac{M_{I}}{T}\right)\right] \varrho_{I J}\left[\frac{E_{J \alpha}}{c^{2} T}-\frac{1}{c} P_{\alpha \nu} \nabla^{\nu}\left(\frac{M_{J}}{T}\right)\right] .
$$


In highest order this equation for non-relativistic systems says that:

$$
\nabla_{t} s+\nabla_{i} j_{S}^{i}=\frac{1}{2 \tau} n \sigma^{i j} \sigma_{i j}+\frac{1}{\tau} z\left(\nabla_{k} v^{k}\right)+\frac{\kappa}{\tau^{2}}\left(\nabla^{i} \tau\right)^{2} .
$$

But if we look at the respective coefficients from the relativistic side:

$$
\begin{aligned}
& n=\frac{\eta}{c} \geq 0, \quad z=\frac{\zeta}{c} \geq 0, \\
& \kappa=\varrho_{I J} \frac{c^{3}}{\mathcal{K}^{2}} \frac{m_{I}^{a} m_{J}^{a}}{T^{2}} \geq 0 .
\end{aligned}
$$

We hence see that the relativistic entropy positivity implies non-relativistic entropy positivity.

Open Access. This article is distributed under the terms of the Creative Commons Attribution License (CC-BY 4.0), which permits any use, distribution and reproduction in any medium, provided the original author(s) and source are credited.

\section{References}

[1] L.D. Landau and E.M. Lifshitz, Fluid mechanics, Pergamon Press, 1997.

[2] N. Banerjee, J. Bhattacharya, S. Bhattacharyya, S. Dutta, R. Loganayagam et al., Hydrodynamics from charged black branes, JHEP 01 (2011) 094 [arXiv:0809.2596] [INSPIRE].

[3] J. Erdmenger, M. Haack, M. Kaminski and A. Yarom, Fluid dynamics of R-charged black holes, JHEP 01 (2009) 055 [arXiv: 0809.2488] [INSPIRE].

[4] D.T. Son and P. Surowka, Hydrodynamics with Triangle Anomalies, Phys. Rev. Lett. 103 (2009) 191601 [arXiv:0906.5044] [INSPIRE].

[5] R. Loganayagam, Entropy Current in Conformal Hydrodynamics, JHEP 05 (2008) 087 [arXiv:0801.3701] [INSPIRE].

[6] S. Bhattacharyya, Entropy Current from Partition Function: One Example, arXiv: 1403.7639 [INSPIRE].

[7] S. Bhattacharyya, Entropy current and equilibrium partition function in fluid dynamics, arXiv: 1312.0220 [INSPIRE].

[8] S. Bhattacharyya, Constraints on the second order transport coefficients of an uncharged fluid, JHEP 07 (2012) 104 [arXiv: 1201.4654] [INSPIRE].

[9] K. Jensen, M. Kaminski, P. Kovtun, R. Meyer, A. Ritz et al., Towards hydrodynamics without an entropy current, Phys. Rev. Lett. 109 (2012) 101601 [arXiv: 1203.3556] [INSPIRE].

[10] K. Jensen, M. Kaminski, P. Kovtun, R. Meyer, A. Ritz et al., Parity-Violating Hydrodynamics in 2+1 Dimensions, JHEP 05 (2012) 102 [arXiv:1112.4498] [INSPIRE].

[11] N. Banerjee, J. Bhattacharya, S. Bhattacharyya, S. Jain, S. Minwalla et al., Constraints on Fluid Dynamics from Equilibrium Partition Functions, JHEP 09 (2012) 046 [arXiv: 1203.3544] [INSPIRE].

[12] N. Banerjee, S. Dutta, S. Jain, R. Loganayagam and T. Sharma, Constraints on Anomalous Fluid in Arbitrary Dimensions, JHEP 03 (2013) 048 [arXiv: 1206.6499] [INSPIRE]. 
[13] M. Rangamani, S.F. Ross, D.T. Son and E.G. Thompson, Conformal non-relativistic hydrodynamics from gravity, JHEP 01 (2009) 075 [arXiv:0811.2049] [INSPIRE].

[14] D.K. Brattan, Charged, conformal non-relativistic hydrodynamics, JHEP 10 (2010) 015 [arXiv: 1003.0797] [INSPIRE].

[15] M. Kaminski and S. Moroz, Non-Relativistic Parity-Violating Hydrodynamics in Two Spatial Dimensions, Phys. Rev. B 89 (2014) 115418 [arXiv:1310.8305] [INSPIRE].

[16] A. Lucas and P. Surówka, Phenomenology of non-relativistic parity-violating hydrodynamics in $2+1$ dimensions, arXiv:1403.5239 [INSPIRE].

[17] M. Rangamani, Gravity and Hydrodynamics: Lectures on the fluid-gravity correspondence, Class. Quant. Grav. 26 (2009) 224003 [arXiv:0905.4352] [INSPIRE].

[18] G. Manfredi, Non-relativistic limits of Maxwell's equations, Eur. J. Phys. 34 (2013) 859 [arXiv:1303.5608]. 